\title{
In vivo adenine base editing of PCSK9 in macaques reduces $L D L$ cholesterol levels
}

\author{
Tanja Rothgangl(1), Melissa K. Dennis², Paulo J. C. Lin², Rurika Oka ${ }^{3}{ }^{3}$, Dominik Witzigmann ${ }^{1}$, \\ Lukas Villiger', Weihong Qi ${ }^{4}$, Martina Hruzova5 ${ }^{5}$ Lucas Kissling ${ }^{1}$, Daniela Lenggenhager6, \\ Costanza Borrelli ${ }^{7}$, Sabina Egli', Nina Frey ${ }^{5}$, Noëlle Bakker ${ }^{1}$, John A. Walker II ${ }^{8}$, Anastasia P. Kadina ${ }^{8}$, \\ Denis V. Victorov ${ }^{8}$, Martin Pacesa ${ }^{1}{ }^{9}$, Susanne Kreutzer ${ }^{4,10}$, Zacharias Kontarakis ${ }^{4,10}$, \\ Andreas Moor ${ }^{7}{ }^{7}$, Martin Jinek ${ }^{9}{ }^{9}$, Drew Weissman ${ }^{11}$, Markus Stoffel ${ }^{(1}{ }^{5}$, Ruben van Boxtel ${ }^{3}$, \\ Kevin Holden ${ }^{8}{ }^{8}$, Norbert Pardi"11, Beat Thöny ${ }^{12,13,14}$, Johannes Häberle ${ }^{12,13,15}$, Ying K. Tam², \\ Sean C. Semple ${ }^{2 \bowtie}$ and Gerald Schwank $\mathbb{B}^{1,5} \bowtie$
}

\begin{abstract}
Most known pathogenic point mutations in humans are $C \bullet G$ to $T \bullet A$ substitutions, which can be directly repaired by adenine base editors (ABEs). In this study, we investigated the efficacy and safety of ABEs in the livers of mice and cynomolgus macaques for the reduction of blood low-density lipoprotein (LDL) levels. Lipid nanoparticle-based delivery of mRNA encoding an ABE and a single-guide RNA targeting PCSK9, a negative regulator of LDL, induced up to $67 \%$ editing (on average, $61 \%$ ) in mice and up to $34 \%$ editing (on average, $26 \%$ ) in macaques. Plasma PCSK9 and LDL levels were stably reduced by $95 \%$ and $58 \%$ in mice and by $32 \%$ and $14 \%$ in macaques, respectively. ABE mRNA was cleared rapidly, and no off-target mutations in genomic DNA were found. Re-dosing in macaques did not increase editing, possibly owing to the detected humoral immune response to $A B E$ upon treatment. These findings support further investigation of ABEs to treat patients with monogenic liver diseases.
\end{abstract}

P rogrammable CRISPR-Cas nucleases enable genome editing by generating double-stranded DNA breaks at the target locus ${ }^{1}$. For introducing precise changes at single-nucleotide resolution, induced DNA breaks require repair by exogenous donor templates via homologous recombination ${ }^{1-4}$. This process, however, is highly inefficient in post-mitotic cells ${ }^{5,6}$, and the use of targeted nucleases for gene editing therapies is, thus, limited to proliferating cells. Base editors are more recently developed genome engineering tools, where either a cytidine or an adenine deaminase is covalently linked to catalytically impaired Cas9. They convert C•T into $\mathrm{T} \bullet \mathrm{A}$ or $\mathrm{A} \bullet \mathrm{T}$ into $\mathrm{G} \bullet \mathrm{C}$ base pairs without the requirement of homology-directed repair and, therefore, enable precise and efficient editing in tissues with slow turnover rates, such as the liver ${ }^{7-10}$. Considering that most pathogenic point mutations are $\mathrm{C} \bullet \mathrm{G}$ to $\mathrm{T} \bullet \mathrm{A}$ conversions, $\mathrm{ABEs}$ are of particular interest for in vivo genome editing therapies ${ }^{8}$.

For clinical application of base editing, the potential generation of off-target mutations represents a major concern. Off-target mutations could be single guide RNA (sgRNA) dependent ${ }^{11,12}$ or sgRNA independent ${ }^{13-15}$ and are influenced by the levels and duration of base editor expression ${ }^{16-18}$. Thus, the risks of generating off-target mutations in therapies are likely to depend on the delivery method and dose. In addition, in vivo base editing has not yet been demonstrated in large animal models, and it remains unclear whether currently available delivery vectors are efficient enough to enable base editing in patients with therapeutic effects. In this study, we investigated the safety and efficacy of in vivo adenine base editing in the liver of mice and non-human primates (NHPs). We targeted PCSK9 (proprotein convertase subtilisin/kexin type 9), which is primarily expressed in the liver and acts as a negative regulator of the LDL receptor ${ }^{19}$. Disrupting its function reduces blood LDL levels and represents a promising therapeutic approach for familial hypercholesterolemia with heterozygous loss-of-function mutations in LDLR or gain-of-function mutations in PCSK9 (refs. ${ }^{20-22}$ ). We report that lipid nanoparticle (LNP)-mediated delivery of ABE-encoding nucleoside-modified mRNA, together with a chemically stabilized sgRNA, enables efficient editing in mice and NHPs without inducing off-target mutations on genomic DNA.

Results

Inactivating PCSK9 by adenine base editing in mouse liver reduces blood LDL. ABEs can be used to eliminate gene function by disrupting canonical splice sites $^{23,24}$. To assess if this approach enables degradation of Pcsk9 mRNA and protein, we targeted several canonical Pcsk9 splice sites in murine Hepal-6 cells using adenine base editing (Supplementary Fig. 1a). The highest editing rates were

'University of Zurich, Institute for Pharmacology and Toxicology, Zurich, Switzerland. ${ }^{2}$ Acuitas Therapeutics Inc., Vancouver, BC, Canada. ${ }^{3}$ Oncode Institute, Princess Máxima Center for Pediatric Oncology, Utrecht, Netherlands. ${ }^{4}$ Functional Genomics Center Zurich, ETH Zurich/University of Zurich, Zurich, Switzerland. ${ }^{5}$ Department of Biology, Institute for Molecular Health Sciences, ETH Zurich, Zurich, Switzerland. ${ }^{6}$ Department of Pathology and Molecular Pathology, University Hospital and University of Zurich, Zurich, Switzerland. 'Department of Biosystems Science and Engineering, ETH Zurich, Zurich, Switzerland. ${ }^{8}$ Synthego Corporation, Redwood City, CA, USA. ${ }^{9}$ Department of Biochemistry, University of Zurich, Zurich, Switzerland. ${ }^{10}$ Genome Engineering and Measurement Laboratory, ETH Zurich, Zurich, Switzerland. "Department of Medicine, University of Pennsylvania, Philadelphia, PA, USA. ${ }^{12}$ Division of Metabolism and Children's Research Centre, University Children's Hospital Zurich, Zurich, Switzerland. ${ }^{13}$ Zurich Center for Integrative Human Physiology, Zurich, Switzerland. ${ }^{14}$ Neuroscience Center Zurich, Zurich, Switzerland. ${ }^{15}$ Institute of Molecular Life Sciences, University of Zurich, Zurich, Switzerland. 凶e-mail: ssemple@acuitastx.com; schwank@pharma.uzh.ch 
observed at the GT splice donor site of murine Pcsk9 intron 1 using sgRNA_mP01 ( $84 \pm 4.6 \%$; Supplementary Figs. 1b,c and 2a-c), leading to a substantial reduction in Pcsk9 mRNA and protein levels (Supplementary Fig. 2d,e). For the human Pcsk9 splice donor site of intron 1, we observed similar editing efficiencies with the corresponding sgRNA in human HepG2 cells $(89 \pm 1.6 \%$; Supplementary Fig. $2 \mathrm{f}-\mathrm{h})$, and, because the target sequence is perfectly conserved to NHPs (Supplementary Fig. 2b), we selected sgRNA_mP01 and sgRNA_hP01 for our in vivo experiments in mice and cynomolgus macaques, respectively (Fig. 1a).

In recent years, $\mathrm{ABEs}$ with different TadA and Cas9 variants have been established ${ }^{25}$. To first identify an efficient ABE variant for editing our target locus in the liver, we delivered different SpCas9-TadA constructs together with sgRNA_mP01 to the liver of 5-week-old C57BL/6J mice using a dual adeno-associated virus (AAV) inteinsplit system ${ }^{26}$ (Fig. 1b and Supplementary Fig. 3a). Six weeks after AAV-based ABE delivery, genomic DNA was isolated from the liver, and the target locus was analyzed by targeted amplicon deep sequencing. The highest editing rates $(60 \pm 18 \%$; Fig. 1c) were obtained with ABEmax, in which laboratory-evolved TadA7.10 is N-terminally fused to nickase (n)SpCas9. Phenotypic analysis of these mice also revealed a significant decrease in plasma Pcsk9 levels (from $365 \pm 38 \mathrm{ng} \mathrm{ml}^{-1}$ to $53 \pm 9 \mathrm{ng} \mathrm{ml}^{-1}$ ) as well as plasma LDL levels (from $1.53 \pm 0.14 \mathrm{mmol} \mathrm{L}^{-1}$ to $0.46 \pm 0.09 \mathrm{mmol} \mathrm{L}^{-1}$ ) (Fig. 1d,e). Mice treated with TadA N-terminally fused to nuclease dead (d)SpCas9 obtained less editing (21.5 $\pm 5.1 \%$; Fig. 1c), and exchanging TadA7.10 with the hyperactive TadA $8 \mathrm{e}^{27}$ increased editing rates on the bystander adenine, but not editing of the target adenine, in the splice donor site (Fig. 1c and Supplementary Fig. 3d). In addition, identified insertion/deletion (indel) mutations at the target site were lower in ABEmax-treated versus ABE8e-treated mice $(0.027 \pm 0.015 \%$ versus $0.276 \pm 0.057 \%$; Fig. $1 c)$. Together, these findings prompted us to continue all further in vivo experiments in this study with ABEmax.

Transient base editing by LNP-mediated mRNA/sgRNA delivery. In tissues with a slow turnover rate, AAV-mediated delivery leads to long-term transgene expression ${ }^{28}$ (Supplementary Fig. 3b,c). For the 'hit-and-run' process of genome editing, this is neither necessary nor desired, as modifications in genomic DNA are permanent. Moreover, prolonged $\mathrm{ABE}$ expression could lead to an accumulation of off-target mutations over time and might induce immune responses and a rejection of cells expressing bacterial Cas9 or TadA. Corroborating the latter concern, we found specific antibodies against $S p C a s 9$ and TadA in AAV-treated mice, which is in line with a recent study that detected humoral and $\mathrm{T}$ cell responses in mice where $\mathrm{SaCas} 9$ was delivered via $\mathrm{AAV}^{29}$ (Fig. 1f). Therefore, we next employed LNPs to transiently deliver ABEmax mRNA and sgRNA_ mP01 into the mouse liver. 1-methoxyuridine-modified ABEmax mRNA was co-formulated with chemically modified sgRNAs ${ }^{30,31}$ in a 1:1 molecular weight ratio into LNPs (Supplementary Fig. 4a) and systemically administrated into $\mathrm{C} 57 \mathrm{BL} / 6 \mathrm{~J}$ mice via tail vein injection. A single injection dose of $1 \mathrm{mg} \mathrm{kg}^{-1}, 1.5 \mathrm{mg} \mathrm{kg}^{-1}$ and $3 \mathrm{mg} \mathrm{kg}$ resulted in a mean editing efficiency of $3.9 \pm 2.8 \%, 12.9 \pm 5.5 \%$ and $50.9 \pm 8.8 \%$, respectively (Fig. $1 \mathrm{~g}$ ). No significant difference in editing rates was observed between the two different variants of chemically modified sgRNAs (Supplementary Fig. 4a,b). Re-dosing after 2 $\mathrm{d}$ in the $1.5 \mathrm{mg} \mathrm{kg}^{-1}$ and $3 \mathrm{mg} \mathrm{kg}^{-1}$ groups further increased editing rates to $39.9 \pm 10 \%$ and $67.3 \pm 18 \%$, respectively, as measured from whole liver lysates (Fig. $1 \mathrm{~g}$ and Supplementary Fig. 4c,d), and phenotypic analysis revealed substantial reduction in plasma Pcsk9 lev-

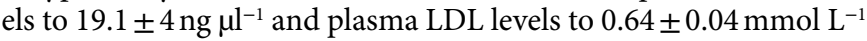
(Fig. 1h,i and Supplementary Fig. 4e). Although LNP administration led to a temporary increase in serum transaminases, indicative of hepatocellular injury, mice were otherwise asymptomatic (Supplementary Fig. 4f,g), and neither Cas9- nor TadA-specific antibodies were detected 2.5 weeks or 15 weeks after treatment (Fig. 1j). As expected, LNPs also showed high affinity for delivery to hepatocytes; editing rates were increased to $86.9 \pm 1.9 \%$ when genomic DNA was isolated from primary hepatocytes as compared to whole liver lysates, where $30 \%$ of cells are non-parenchymal ${ }^{32}$; in other organs, editing rates remained below $7 \%$ (testes, spleen, lung, heart, kidney, diaphragm and skeletal muscle; Supplementary Fig. 4h). As intended, we also observed that LNP-mediated mRNA/ sgRNA delivery leads to transient expression and activity of the gene editing machinery: ABE mRNA levels peaked at $12 \mathrm{~h}$ after delivery and quickly declined thereafter; Pcsk9 mRNA levels rapidly declined within the first $24 \mathrm{~h}$; and editing rates reached a plateau after $48 \mathrm{~h}$ (Fig. 2a,b).

Molecular assessment of off-target editing in RNA. The generation of off-target mutations could limit clinical application of base editing. Off-target mutations can be either sgRNA dependent $^{11,12}$ or independent of the sgRNA sequence and triggered by the promiscuous reactivity of deaminases in the mRNA or genomic $\mathrm{DNA}^{13-15}$. Because recent in vitro studies in human cell lines demonstrated that $\mathrm{ABE}$ expression causes tens of thousands of sgRNA-independent A-to-G transitions in mRNA ${ }^{13,15,33}$, we first assessed transcriptome-wide off-target deamination after in vivo $A B E$ delivery using RNA sequencing (RNA-seq) (on average, 160 million reads per library). Although we were able to confirm previous in vitro studies and found that transfection of ABEmax-expressing plasmids into HEK293T cells induced, on average, more than 11,000 A-to-G transitions on the transcriptome $^{13,15}$ (Fig. 2c and Supplementary Fig. 5a,b), neither LNP- nor $\mathrm{AAV}$-mediated delivery of ABE led to a major increase in A-to-G transitions in the transcriptome of hepatocytes isolated from treated mice (on average, 44 and 20 A-to-G transitions, respectively; Fig. 2c). Moreover, off-target effects in LNP-treated animals were temporary and no longer detectable at Day 17 after injection (Fig. 2c). Previous studies suggested that unguided deamination by base editors is dose dependent ${ }^{16-18}$, providing a possible explanation for the low rates of off-target deamination in mRNA in vivo. Indeed, when we compared $\mathrm{ABE}$ expression levels in vitro and in vivo, we found that expression in transfected HEK293T cells was in the order of 3-4 magnitudes higher than in hepatocytes isolated from LNP- or AAV-treated mice (Fig. 2c).

Molecular assessment of off-target editing in DNA. In contrast to off-target mutations in RNA, off-target mutations in genomic DNA are permanent. Thus, the potential of ABEs to induce DNA off-target edits is an important consideration for clinical risk assessment of transient base editing approaches. Recent ex vivo studies in human induced pluripotent stem cells, two-cell stage mouse embryos and rice callus cells associated cytidine base editor (CBE) expression, but not $\mathrm{ABE}$ expression, with sgRNA-independent off-target deamination in genomic $\mathrm{DNA}^{34-36}$. To assess whether this observation holds true for in vivo adenine base editing in the liver, we analyzed the genomic DNA of hepatocytes from AAV- and LNP-treated animals by whole-genome sequencing (WGS). Considering that unguided deamination is likely to occur in a random fashion at different sites in each cell, and taking into account that all next-generation sequencing (NGS) technologies suffer from baseline error rates $^{37}$, mutations would be overshadowed by noise if bulk DNA from a pool of cells is sequenced. Therefore, we isolated primary hepatocytes from treated animals and expanded them ex vivo ${ }^{38}$ to obtain enough clonal DNA for WGS at 30× coverage (Supplementary Fig. 6a). Clones without editing at the target locus were excluded, ensuring that only hepatocytes that were exposed to ABE were analyzed. Consistent with previous ex vivo studies, we found no significant increase in $A>G$ transitions in clones from LNP- or AAV-treated mice (Fig. 3a), and, also, the relative contribution of $A>G$ transitions 
a Mus musculus

CCGCCGTTGCTCCAAGGTATGGG GGCGGCAACGAGGTTCCATACCC

Macaca fascicularis/Homo sapiens CCACCGCTGCGCCAAGGTGCGGG GGTGGCGACGCGGTTCCACGCCC

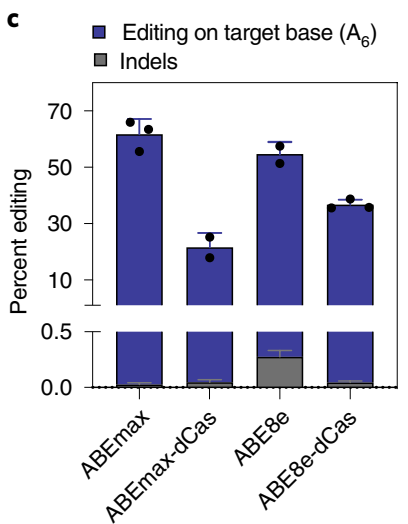

g Editing on target base $\left(\mathrm{A}_{6}\right)$ $\square$ Indels

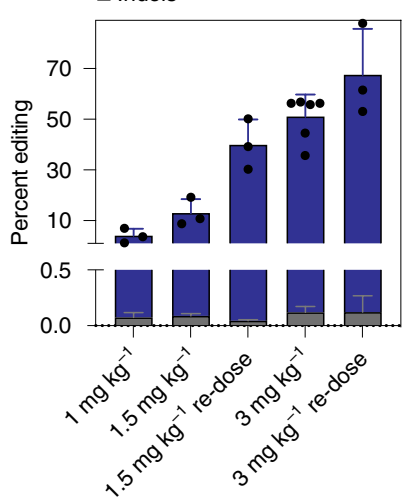

b

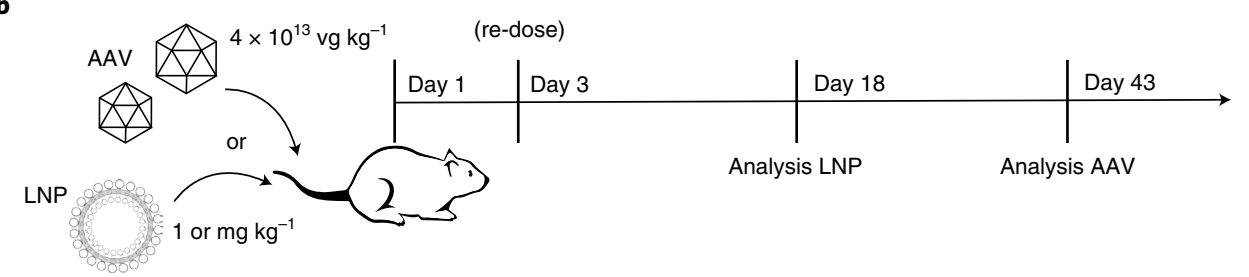

d

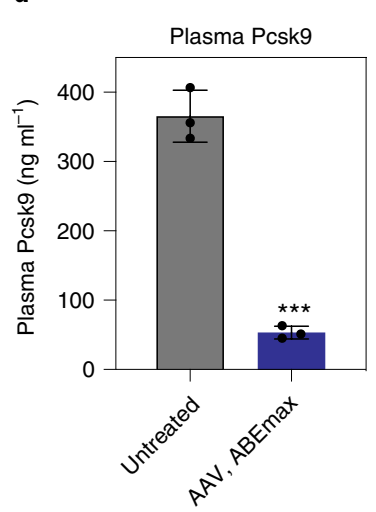

h

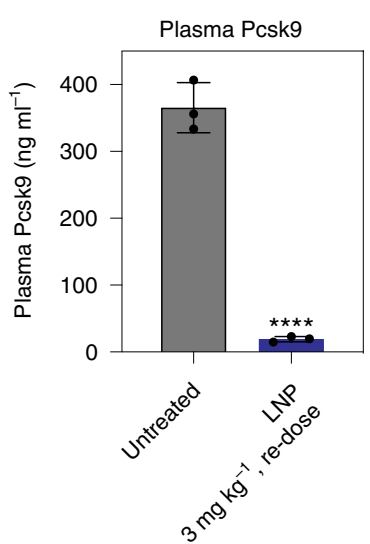

e

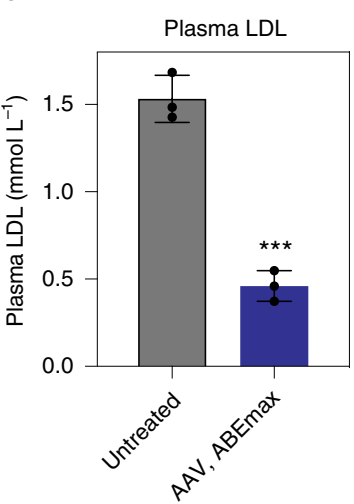

i

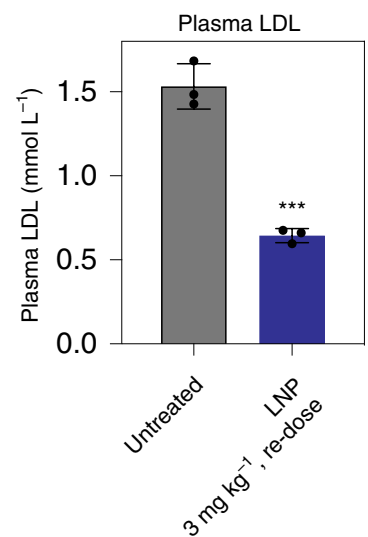

f

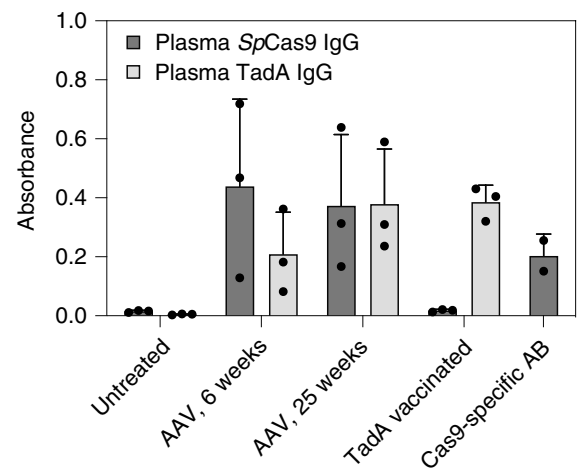

j

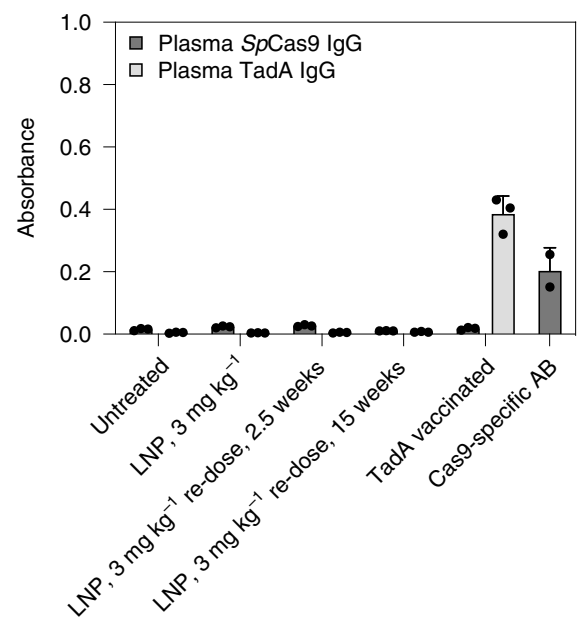

Fig. 1 | In vivo adenine base editing of the Pcsk9 locus in the mouse liver. a, sgRNA target sequences in the mouse and macaque/human PCSK9 gene. The exonic region (exon 1) is highlighted in red; the intronic region is highlighted in black. The GT splice donor recognition site is highlighted in bold letters. The NGG-PAM site is indicated in blue. $\mathbf{b}$, Schematic outline of the mouse experiments. Vectors were administered intravenously via tail vein injection in adult C57BL/6J mice. c, Percent editing after treatment with different ABE variants. Insertions and deletions are summarized as 'Indels'. Values represent mean \pm s.d. of $n=3, n=2, n=2$ and $n=3$ animals. d, Plasma Pcsk9 protein levels as determined by ELISA. ${ }^{\star \star \star} P=0.0002$. e, Plasma LDL cholesterol levels. ${ }^{\star \star \star} P=0.0003$. f, Background-subtracted absorbance $\left(A_{450}-A_{540}\right)$ representing the relative amount of anti-Cas9- or anti-TadA-specific plasma IgG antibodies as determined by ELISA. Positive control: plasma of TadA vaccinated animals or commercial Cas9-specific antibody at a concentration of $200 \mathrm{ng} \mathrm{ml}^{-1}(n=2)$. $\mathbf{g}$, Percent editing after treatment with different doses of LNP-encapsulated mRNA/sgRNA. Insertions or deletions are summarized as 'Indels'. h, Plasma Pcsk9 protein levels as determined by ELISA. ${ }^{\star \star \star \star} P<0.0001$. i, Plasma LDL cholesterol levels. ${ }^{\star \star \star} P=0.0004$. j, Background-subtracted absorbance $\left(A_{450}-A_{540}\right)$ representing the relative amount of anti-Cas9- or anti-TadA-specific plasma antibodies as determined by ELISA. Positive control: plasma of TadA vaccinated animals or commercial Cas9-specific antibody at a concentration of $200 \mathrm{ng} \mathrm{ml}^{-1}(n=2)$. Unless otherwise stated, values represent mean \pm s.d. of $n=3$ animals. Means were compared using two-tailed unpaired $t$-tests. vg, vector genomes.

to total single-nucleotide variants (SNVs) was not increased (Fig. 3b and Supplementary Fig. 6b,c). In addition, cosine similarity analysis revealed that clones derived from AAV- or LNP-treated mice showed no enrichment in TadA trinucleotide signatures ${ }^{39}$ compared $^{2}$ to negative control clones (derived from untreated and CBE-treated mice) (Fig. 3c and Supplementary Fig. 6b,c).

Base editor off-target mutations in genomic DNA could also be sgRNA dependent, induced by the recruitment of the
Cas9-deaminase complex to sequences similar to the target locus ${ }^{11,12}$. Therefore, we next investigated the target specificity of sgRNA_ $\mathrm{mP01}$ and performed circularization for in vitro reporting of cleavage effects by sequencing (CIRCLE-seq) and circularization for high-throughput analysis of nuclease genome-wide effects by sequencing (CHANGE-seq) analysis ${ }^{40,41}$ in genomic mouse DNA treated in situ with SpCas9 nuclease complexed with sgRNA_mP01 (Fig. 3d and Supplementary Fig. 7). Subsequent amplicon sequencing 
a
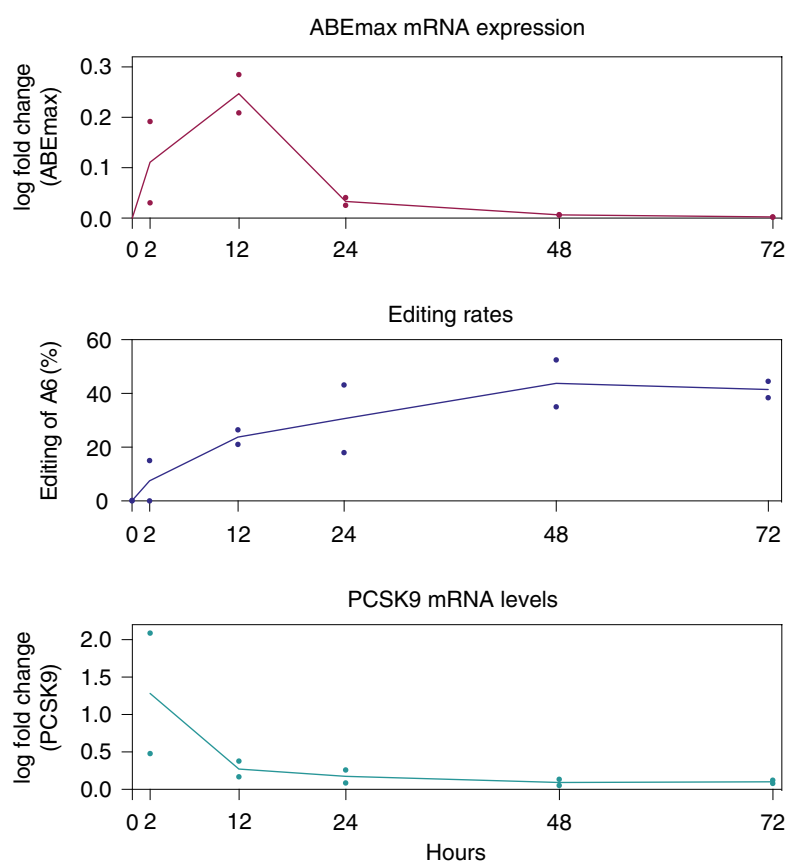

b
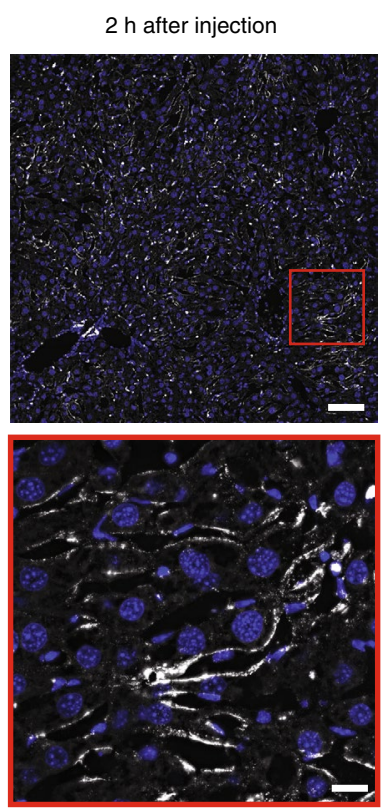

$12 \mathrm{~h}$ after injection
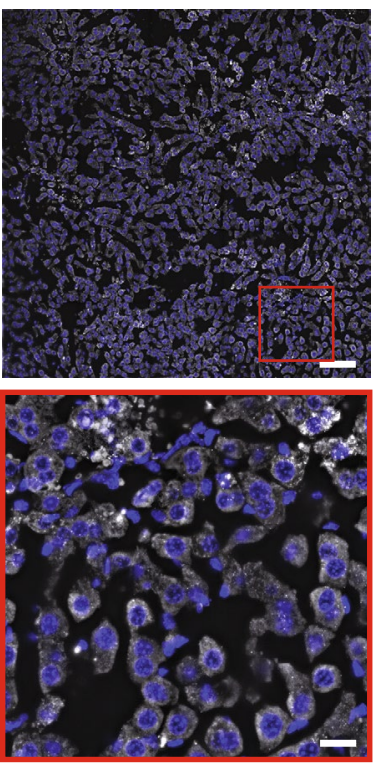

C

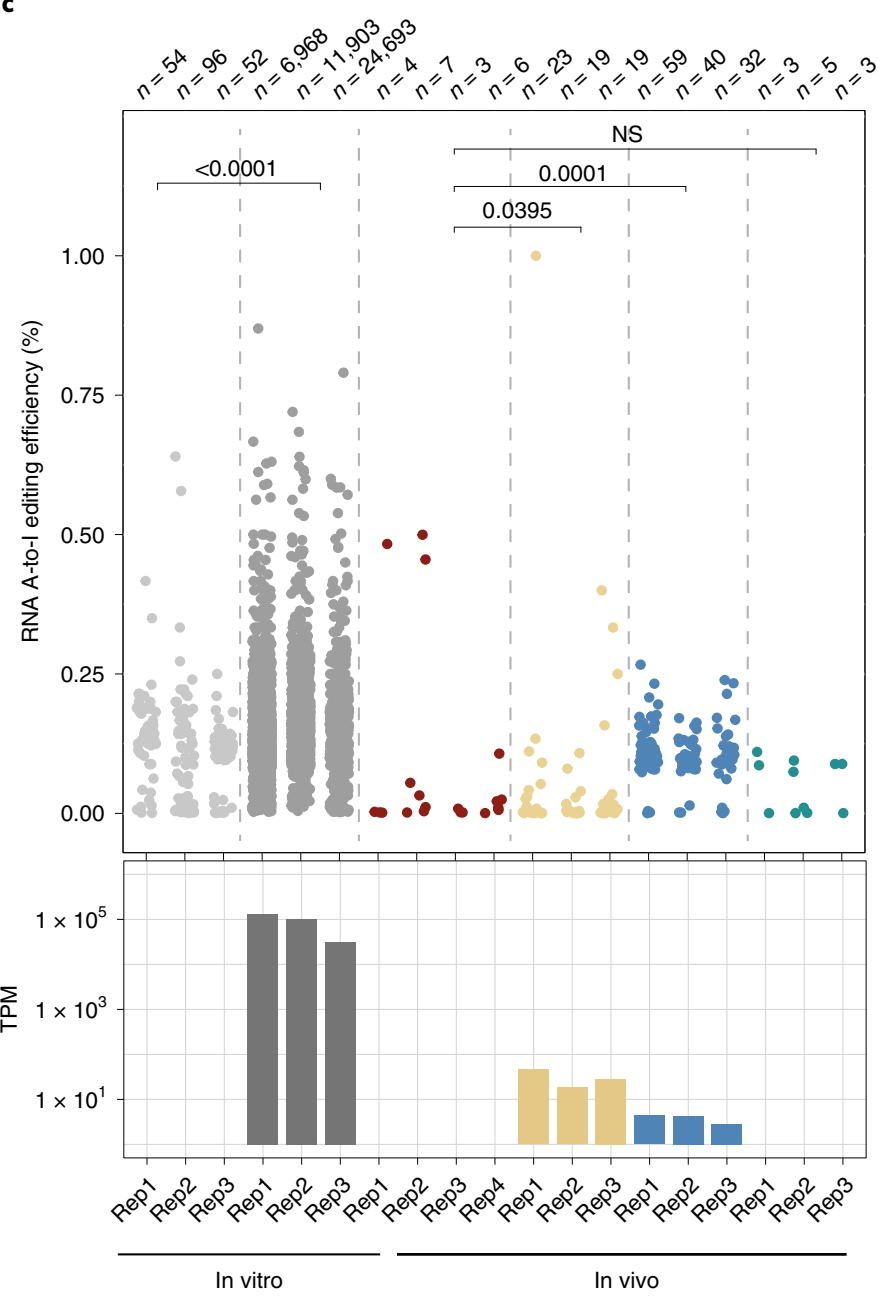

Untransfected, HEK293T

ABEmax transfected, HEK293T

Untreated

AAV, 6 weeks, primary hepatocytes

LNP, $48 \mathrm{~h}$, primary hepatocytes

LNP, 17 d, primary hepatocytes

Fig. 2 | LNP-mediated ABE mRNA delivery leads to transient base editing without inducing substantial off-target deamination in the transcriptome. a, LNPs co-formulated with ABE mRNA and sgRNA_mP01 were systemically delivered. The top panel depicts ABEmax mRNA expression assessed by RT-qPCR. The middle panel depicts editing rates assessed by Sanger sequencing. The bottom panel depicts Pcsk9 mRNA levels assessed by RT-qPCR. The line represent mean of $n=2$ animals per time point. b. Expression and localization of ABEmax mRNA $2 \mathrm{~h}$ or $12 \mathrm{~h}$ after injection of $3 \mathrm{mg} \mathrm{kg}^{-1} \mathrm{LNP}_{\text {assessed by }}$ smFISH in the liver. Twelve hours after injection, mRNA is predominantly cytoplasmatic. Blue, DAPI; white, ABEmax mRNA. Scale bar, top panel, $100 \mu \mu$; scale bar, bottom panel, $20 \mu \mathrm{m}$. smFISH was performed once. c, Top, RNA-wide A-to-I editing assessed by whole-transcriptome sequencing. Each dot represents one editing event. The total number of editing events is indicated above. Each lane represents one individual biological replicate per animal. In vitro RNA-seq data are from HEK293T cells that were co-transfected with plasmids expressing ABEmax and sgRNA_mP01. Means of all replicates per sample were compared using one-tailed unpaired $t$-test for ex vivo samples and one-way ANOVA for in vivo samples. Bottom, telative TadA transcript expression in transcripts per million (TPM). NS, not significant.

$(>10,000$ reads) of the top ten identified potential off-target sites in the liver of AAV- or LNP-treated animals showed no significant increase in A-to-G conversions or indel mutations compared to untreated control animals (Fig. 3d and Supplementary Table 1). Taken together, we found no evidence for the generation of substantial off-target mutations in genomic DNA after AAV- or 
LNP-mediated adenine base editing in vivo. Moreover, we did not observe malignant transformations in the livers of $\mathrm{AAV}$ - or LNP-treated mice after 18 and 25 weeks, and prolonged ABE expression over 1 year in mice sensitized to tumor development by liver-specific $\operatorname{Trp} 53$ deletion $^{42,43}$ did not enhance hepatocellular carcinoma (HCC) formation (Fig. 3e,f and Supplementary Fig. 8a-c). Together, these results suggest that adenine base editing in vivo is effective and safe and could potentially be used for therapeutic purposes in patients with genetic liver diseases.

PCSK9 inhibition by adenine base editing in macaques reduces blood LDL. To next assess the feasibility of LNP-mediated adenine base editing in a clinically relevant large animal model, we edited PCSK9 in adult cynomolgus macaques (Macaca fascicularis). Chemically modified sgRNA_hP01 (variant P1, Synthego; Supplementary Fig. 4a) was co-formulated with 1-methoxyuridine-modified ABEmax mRNA into LNP in a 1:1 weight ratio. Four groups of cynomolgus macaques, with three animals per group, received intravenous 1 -h infusions of $0.75 \mathrm{mg} \mathrm{kg}^{-1}$ RNA (low dose) or $1.5 \mathrm{mg} \mathrm{kg}^{-1}$ RNA (high dose) on Day 1. Each dose level was given either as a single dose or as two doses (repeat dose) using a 2-week interval between doses (Fig. 4a). All animals were euthanized on Day 29, and tissues from different organs were collected. The administration of LNP-encapsulated ABE mRNA and sgRNA_hP01 was well tolerated in all macaques of the four dosing groups, and no animal had to be excluded during the study. Although serum transaminases (aspartate transaminase (AST) and alanine transaminase (ALT)) and a panel of pro-inflammatory cytokines and immune-modulating chemokines were transiently increased after dosing, elevated levels had resolved by $7 \mathrm{~d}$ and $24 \mathrm{~h}$, respectively (Fig. 4b-d and Supplementary Figs. 9 and 10). No cumulative effects with repeated dosing, and no further abnormalities in blood chemistry, were observed (Supplementary Fig. 9). Moreover, a histological examination of livers at Day 29 of high-dose animals (single and repeat dose at $1.5 \mathrm{mg} \mathrm{kg}^{-1}$ ) indicated only sporadic and very mild lobular mixed cell infiltration (Fig. 4e). Next, we assessed on-target editing at the splice donor site of PCSK9 intron 1. We extracted genomic DNA from postmortem biopsies of all four liver lobes (six samples per animal; Supplementary Table 4) and performed targeted amplicon deep sequencing. Although a single dose of $0.75 \mathrm{mg} \mathrm{kg}^{-1}$ led only to $2.03 \pm 0.85 \%$ editing, a single dose of $1.5 \mathrm{mg} \mathrm{kg}^{-1}$ resulted in $27.6 \pm 5.87 \%$ A-to-G editing of the target base, with very low indel rates (up to $0.27 \%$; Fig. $4 f$ ). Re-dosing after 2 weeks did not further increase editing, with editing rates remaining at $3.31 \pm 1.73 \%$ in the $0.75 \mathrm{mg} \mathrm{kg}^{-1}$ group and $24.14 \pm 1.52 \%$ in the $1.5 \mathrm{mg} \mathrm{kg}^{-1}$ group (Fig. 4f). Notably, in high-dose animals, we observed a significant reduction in serum PCSK9 levels $(26 \%$ and $39 \%$ reduction from the baseline in the single-dose group and repeat-dose group, respectively; Fig. 4g), which was associated with a lowering of serum LDL levels (9\% and 19\% reduction from the baseline in the single-dose group and repeat-dose group, respectively; Fig. 4h).

Humoral immune response upon $\mathrm{ABE}$ treatment in macaques. In contrast to LNP-mediated adenine base editing in mice, re-dosing in macaques did not lead to a further increase in editing (Fig. 4f). A possible explanation for this finding is that the stimulation of a pro-inflammatory environment together with the expression of bacterial $\mathrm{ABE}$ components in the liver evoked a host response during initial treatment. This prompted us to test the serum of treated macaques for SpCas9- and TadA-specific antibodies by enzyme-linked immunosorbent assay (ELISA). When we first analyzed the $0.75 \mathrm{mg} \mathrm{kg} \mathrm{kg}^{-1}$ and $1.5 \mathrm{mg} \mathrm{kg}^{-1}$ single-dose groups, we observed only a minor and statistically not significant increase in absorbance $\left(A_{450}-A_{540}\right)$ at Day 29 compared to pre-treatment (Day -1; Fig. 4i,j). However, in both repeat-dose groups, we detected SpCas9- and TadA-specific IgG antibodies at Day 29 (Fig. 4i,j). Given the high concordance between cellular and humoral immunoreactivity ${ }^{44}$, we speculate that LNP-treated animals also developed an ABE-specific $\mathrm{T}$ cell response, which might have cleared transfected hepatocytes during re-dosing. If this hypothesis is true, earlier re-dosing before the formation of adaptive immunity or transient immunosuppression might further increase editing rates.

Off-target editing analysis in macaques. High tissue tropism of the delivery vector and high target specificity of the sgRNA are essential criteria for the safety of genome editing therapies. To assess hepatotropism of the applied LNP formulation, we analyzed on-target editing rates in nine different organs from animals of the high-dose groups. We found that editing rates in testes, brain, skeletal muscle, pancreas, lung, kidney, heart and adrenal gland remained below $1 \%$. Only in the spleen we observed editing rates of $6.1 \pm 2.7 \%$ and $12.4 \pm 7.8 \%$ in single- and repeat-dose treated animals, respectively (Fig. 4k). These results are in line with a recent study that demonstrated efficient internalization of LNP-mRNA complexes in macaques by antigen-presenting cells ${ }^{45}$. To next address target specificity of sgRNA_hP01, we identified candidate off-target sites in the human genome. We performed CIRCLE-seq and CHANGE-seq ${ }^{35,36}$ on human genomic DNA isolated from HEK293T cells treated in situ with SpCas9 nuclease and sgRNA_hP01 and iGUIDE ${ }^{46}$ on HEK293T cells transfected with SpCas9 and sgRNA_hP01 (Fig. 41, Supplementary Fig. 11a-c and Supplementary Table 2). To also identify candidate off-target sites specific to the genome of $M$. fascicularis, we additionally performed CHANGE-seq with sgRNA_hP01 on genomic DNA

Fig. 3 In vivo adenine base editing of Pcsk9 does not induce substantial off-target mutations in DNA. a, Total number of A-to-G (including T-to-C) edits per genome. Clones from untreated control $(n=3)$, LNP-treated $(n=6)$ and AAV-treated $(n=6)$ mice. Box plots are standard Tukey plots. The centerline represents the median; the lower and upper hinges represent the first and third quartiles; and the whiskers represent $\pm 1.5 \times$ the interquartile range. Two-tailed unpaired $t$-tests were used to compare means. $\mathbf{b}$, Relative contributions of single-base substitutions in clones shown in $\mathbf{a}$. Values represent mean \pm s.d. Two-tailed unpaired $t$-tests were used to compare means. c, Heat map showing the cosine similarity of mutational signatures of control clones (untreated and CBE-treated) and ABE-treated clones (LNP and AAV) to a predetermined TadA signature ${ }^{39} .1$ (match) and $\mathrm{O}$ (no similarity). For details on the clones, see Supplementary Fig. 6. In positive control clones, a TadA-specific trinucleotide mutation pattern ${ }^{39}$ was computationally added.

d, sgRNA-dependent off-target sites of sgRNA-mP01 identified by CIRCLE-seq. The top ten off-target sites were analyzed by NGS in Hepa1- 6 cells 5 d after transfection with plasmids encoding ABEmax and sgRNA_mP01 (in vitro) and in primary hepatocytes isolated from LNP-treated mice (3 mg kg-1, re-dose), AAV-treated mice (ABEmax) and untreated C57BL/6J mice. The second off-target site could not be determined (n.d.) owing to repetitiveness of the locus. Values represent the highest $A$-to-G conversion frequency within the protospacer. $n=3$ biological replicates per treatment. e, $A l b-C r e \times \operatorname{Trp} 53^{\text {flox}}$ fllox mice treated with AAVs expressing ABEmax and sgRNA_mP01 5 weeks after birth. Depicted is the HCC-free survival of mice. $n=$ number of animals per group.

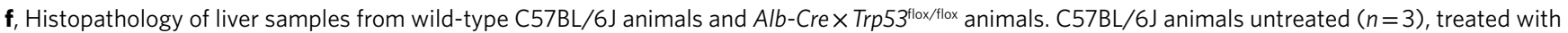
LNP $(n=3)$ or treated with AAV $(n=3)$ were analyzed after 18 or 25 weeks, respectively. Alb-Crex Trp53 floxflox animals untreated ( $n=28)$, treated with control AAV (C-terminal part of the split system and a non-targeting sgRNA) $(n=18)$ or treated with the PCSK9-targeting AAV ( $n=25)$ were analyzed after 1 year. H\&E staining was performed on all animals; three pictures per animal were taken. Representative images are shown. (H\&E; scale bar, $50 \mu \mathrm{m})$. 

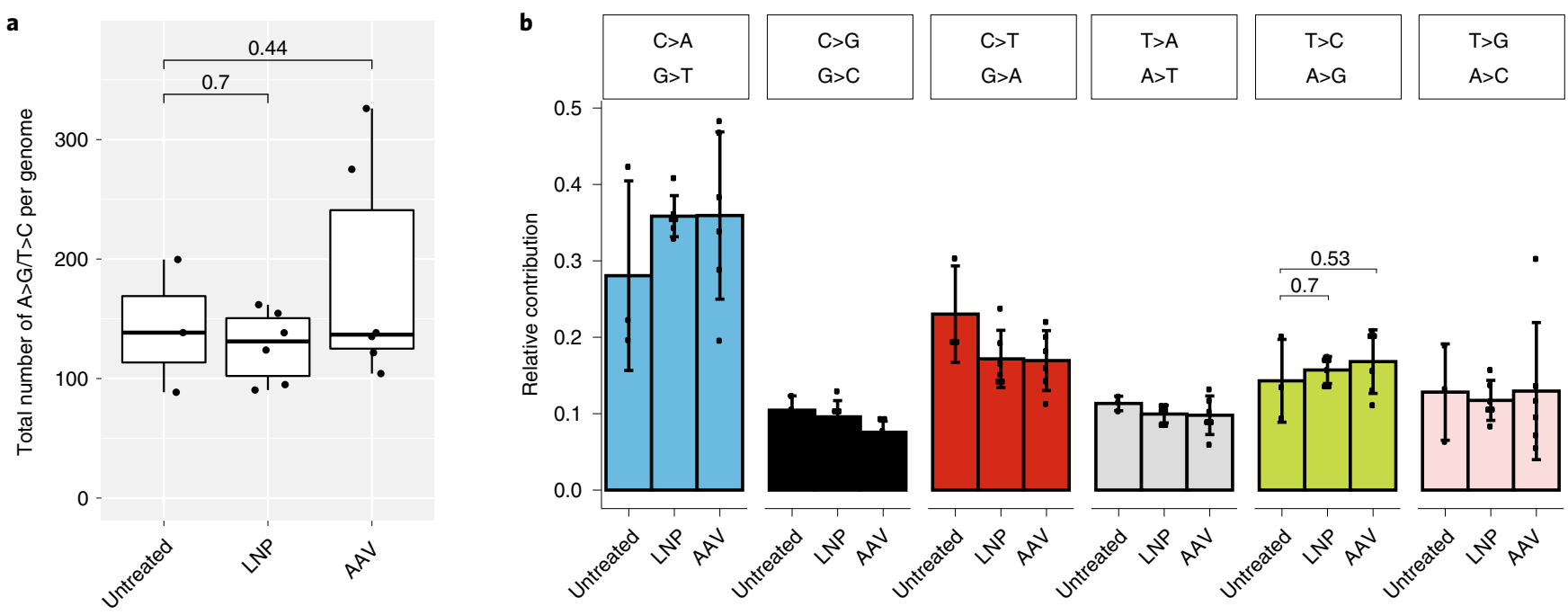

c

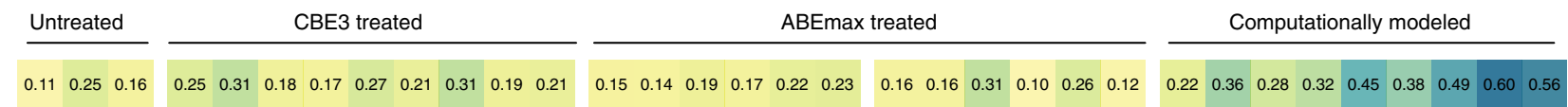

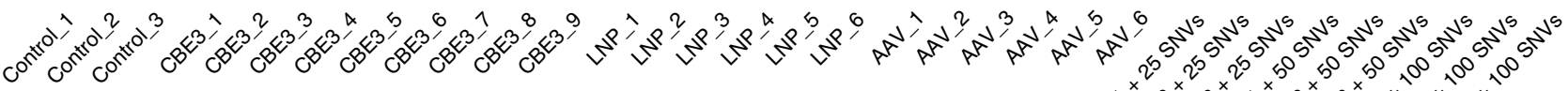

Cosine similarity

$$
0.2
$$

d

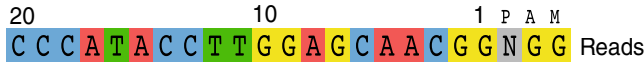

............. C . 984

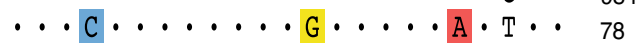

$\mathrm{AG} \cdot \cdots \mathrm{A} \cdot \cdots \cdot \mathrm{A} C \mathrm{C} \cdot \mathrm{T} 48$

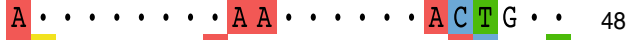

$\mathrm{A} G \cdots \cdots \mathrm{A} \cdots \mathrm{AC} \cdot \mathrm{T}{ }_{46}$

- . . A . - G . T P A . 40

A........ A A A . 36

$\mathrm{T} \mathrm{T} \cdot \cdots \cdot \mathrm{G} \cdot \mathrm{A} G \cdot \cdots\}_{36}$

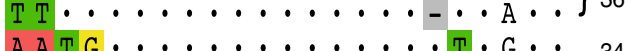

GA.....AA ....A A A . 34

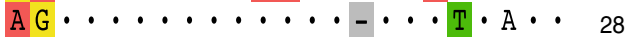
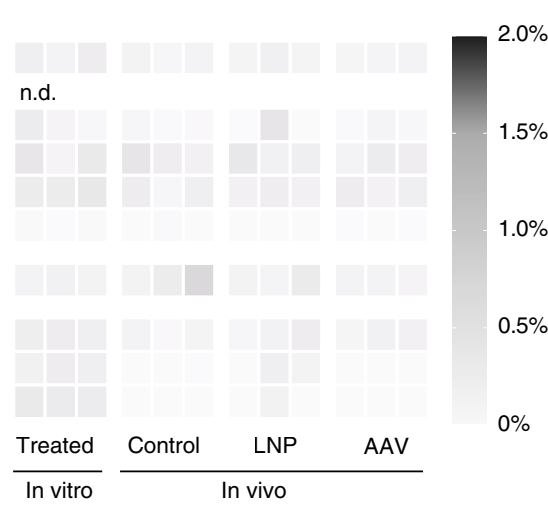

$0 \%$ e

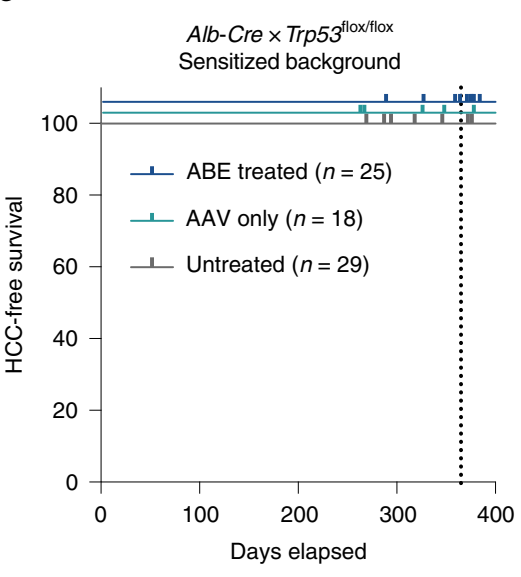

f

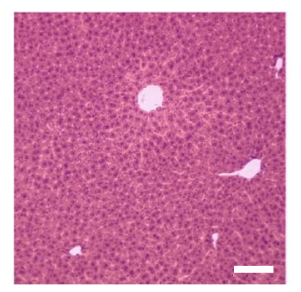

Untreated

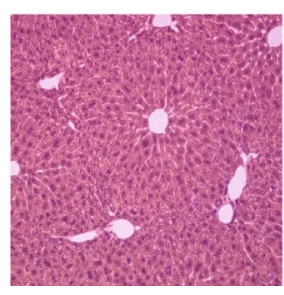

AAV ABE treated

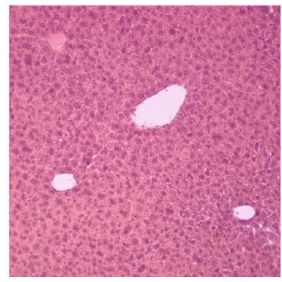

LNP ABE treated

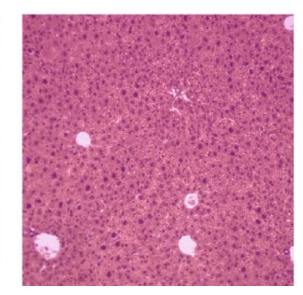

Untreated

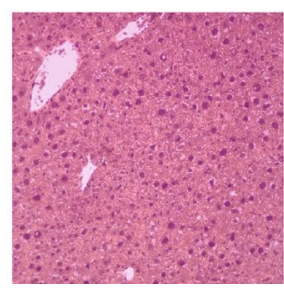

AAV only

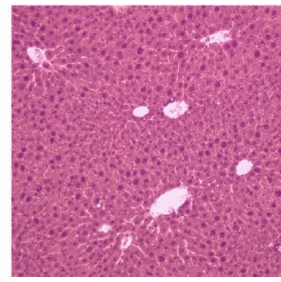

AAV ABE treated

C57BL/6J

Alb-Cre $\times \operatorname{Trp} 53^{\text {flox/flox }}$

and in macaques of the high-dose groups. Despite $89 \%$ on-target editing in transfected HepG2 cells, we did not observe editing above background in any of the eight off-target candidate sites (Fig. 4l). Likewise, in none of the top candidate off-target sites in

isolated from untreated macaques (Supplementary Fig. 11d). The top candidate off-target sites in the human and macaque genome were then analyzed by deep sequencing in human HepG2 cells transfected with plasmids expressing ABEmax and sgRNA_hP01 


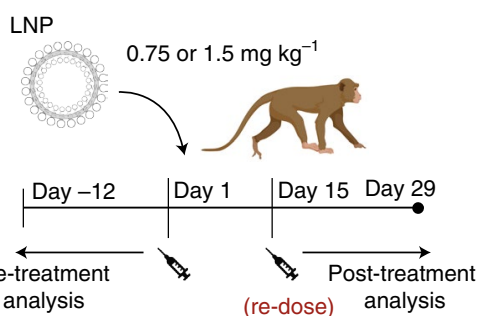

b

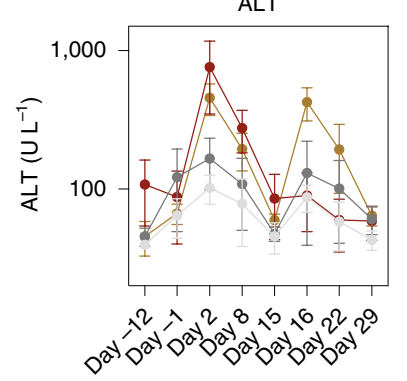

c

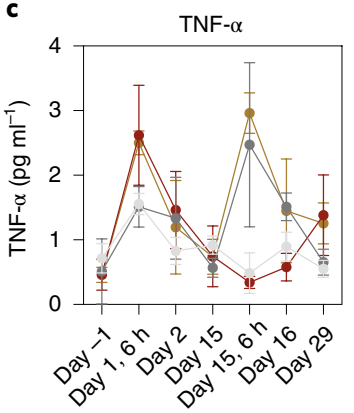

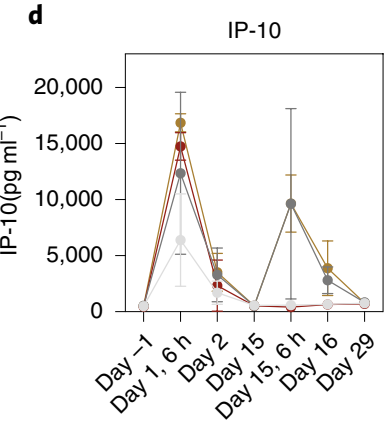

$-1.5 \mathrm{mg} \mathrm{kg}^{-1}$, re-dose
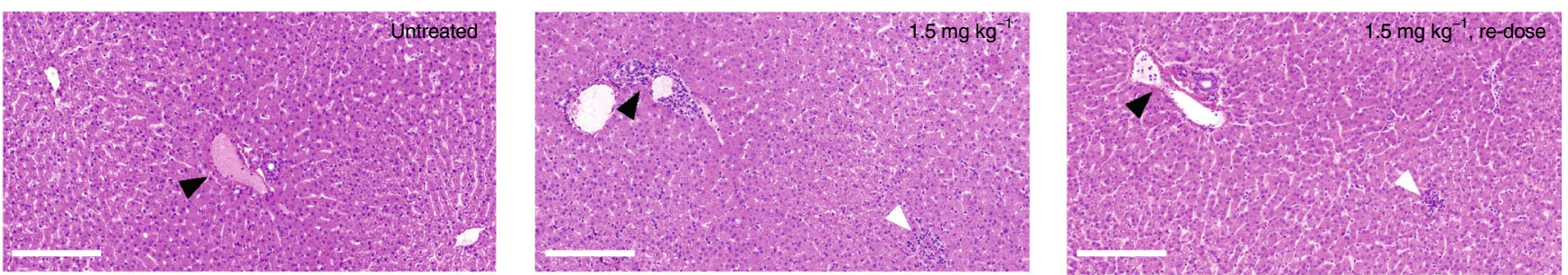

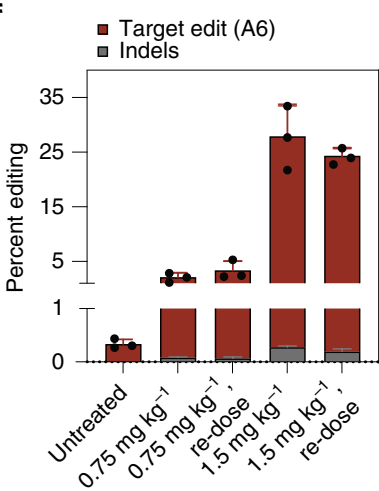

k

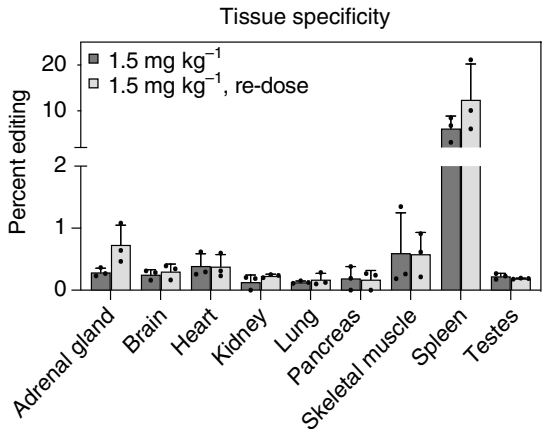

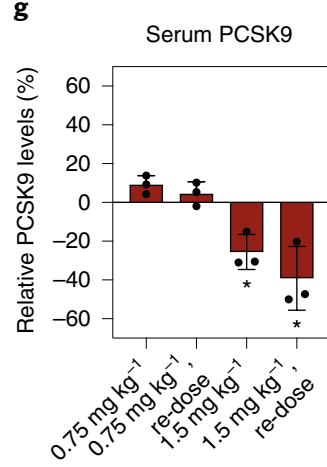

$I_{20}$ h

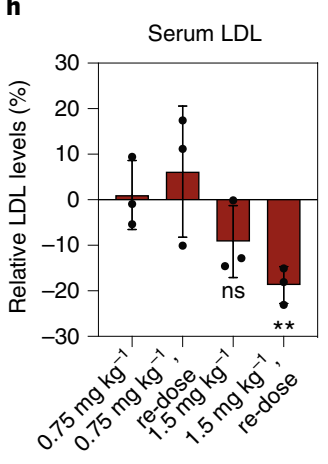

i

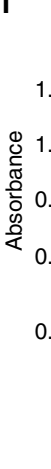

Serum SpCas9 $\lg G \quad \mathbf{j}$

j Serum TadA IgG

\section{C C G C A C C T T G G C G C A G C G G N G G Reads}

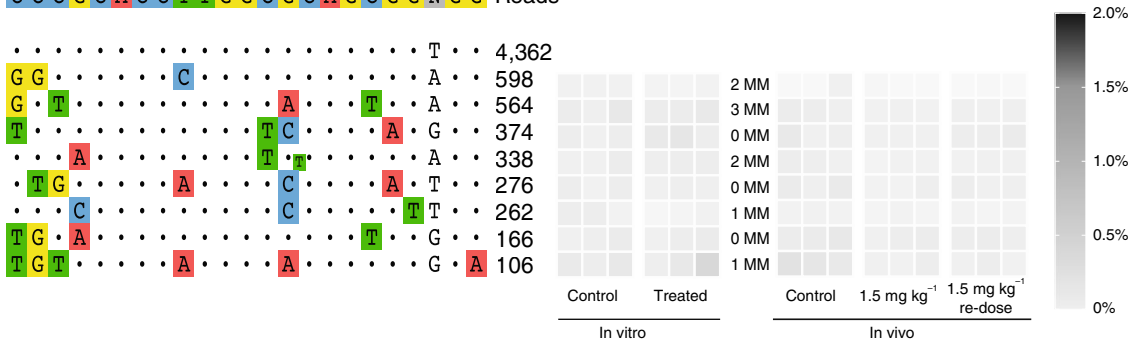

Fig. 4 | In vivo adenine base editing of the PCSK9 locus in the liver of macaques. a, Schematic outline of the experiments. Levels of ALT (b), TNF- $\alpha$ (b) and IP-10 (c). Day 15 is before re-dosing; Day 15, $6 \mathrm{~h}$ is $6 \mathrm{~h}$ after re-dosing. e, Histopathology of liver samples from untreated, $1.5 \mathrm{mg} \mathrm{kg}^{-1}$ single-dosed and $1.5 \mathrm{mg} \mathrm{kg}^{-1}$ re-dosed animals. Three different liver lobes of all animals of the high-dose groups were examined by a trained pathologist. Only very mild lobular mixed inflammatory cell infiltration was observed (white arrowhead). Black arrowheads indicate portal tracts. H\&E; scale bar, $200 \mu$ m. f, Percent editing in treatment groups (six liver biopsies per animal analyzed). g, PCSK9 levels. Serum from two time points before (Day -12 and Day -1 ) and after (Day 22 and Day 29) treatment was analyzed. ${ }^{\star} P=0.020\left(1.5 \mathrm{mg} \mathrm{kg}^{-1}\right) ;{ }^{\star} P=0.027\left(1.5 \mathrm{mg} \mathrm{kg}^{-1}\right.$ re-dose). $\mathbf{h}$, LDL levels. Serum from two time points before (Day -12 and Day -1) and after (Day 22 and Day 29) treatment was analyzed. NS $=0.091 ;{ }^{\star \star} P=0.008$. i, Background-subtracted absorbance $\left(A_{450}-A_{540}\right)$ representing the relative amount of anti-Cas9-specific lgG antibodies. 5\% BSA coating was used to determine background levels. Means were compared to Day $-1 .{ }^{\star} P=0.0095$. j, Background-subtracted absorbance $\left(A_{450}-A_{540}\right)$ representing the relative amount of anti-TadA-specific lgG antibodies. Means were compared to Day $-1 .{ }^{\star} P=0.0336,{ }^{\star \star} P=0.0026$. k, Editing rates in DNA isolated from other tissues than the liver. I, sgRNA-dependent off-target sites of sgRNA_hP01 in the human genome identified using CIRCLE-seq. The top eight hits with orthologous sites in M. fasciularis were analyzed by NGS in vitro (HepG2 cells transfected with plasmids encoding ABEmax and sgRNA_hP01) and in vivo ( $3 \mathrm{mg} \mathrm{kg}^{-1}$ single-dosed and re-dosed). Values represent the highest A-to-G conversion frequency within the protospacer. $n=3$ biological replicates per treatment. Control: DNA isolated from untreated HepG 2 cells and macaque blood cells before treatment. MM, mismatches between the human and M. fasciularis genome. Values represent mean $\pm \mathrm{s}$.d. of $n=3$ animals. Means were compared using one-tailed paired $t$-test. 
treated macaques was substantial A-to-G editing observed (Fig. 41 and Supplementary Fig. 11d).

\section{Discussion}

Base editors are widely applied genome editing tools that hold great potential for therapeutic applications. They enable correction of all four transition mutations, which account for approximately $30 \%$ of annotated pathogenic mutations. Although protospacer adjacent motif (PAM) availability restricts the targeting scope of classical SpCas9 base editors, this limitation can theoretically be circumvented by the use of base editors that are built on novel Cas variants, making the vast majority of pathogenic transition mutations targetable ${ }^{25}$. Supporting this hypothesis, a recent study reported correction of over 3,000 disease-associated SNVs with more than $90 \%$ precision using different base editor variants ${ }^{47}$. In this study, we applied base editing to install a splice site mutation in PCSK9, and we show here that LNP-mediated delivery of ABE-encoding nucleoside-modified mRNA, together with chemically modified sgRNA, results in editing rates of up to $30 \%$ in the liver of macaques. These efficiencies should be sufficient for therapeutic application in several genetic liver diseases, including urea cycle disorders, phenylketonuria and tyrosinemia ${ }^{48-50}$. Moreover, it is conceivable that adjustment of dose levels and schedules could further increase editing rates in macaques to those observed in mice, making the PCSK9 base editing approach reported here also suitable for treating patients with familial hypercholesterolemia.

One of the major considerations for the clinical development of safe and effective genome editing therapies is the minimization or elimination of off-target editing and mutations. Thus, a therapeutically viable approach should maximize on-target editing but minimize off-target editing. We demonstrate that transient LNP-mediated $\mathrm{ABE}$ delivery into mouse livers enables up to $88 \%$ on-target editing in hepatocytes without inducing substantial sgRNA-dependent or sgRNA-independent (unguided) off-target editing in genomic DNA. Notably, the number of hepatocytes analyzed for unguided off-target deamination in our study was limited, and generation of mutations below our detection limit or in a sub-pool of hepatocytes cannot be excluded. Additional long-term studies in relevant model systems would, therefore, be highly valuable to further assess safety of in vivo adenine base editing. However, it is likely that the risks resulting from off-target editing are relatively low. First, over an average human lifespan, every hepatocyte collects, on average, more than 1,000 spontaneous $\mathrm{SNVs}^{51}$, suggesting that humans can be well capable of tolerating a large number of point mutations. Second, continuous expression of ABEmax over the period of 1 year in Trp53 mutant mice did not increase HCC formation.

In conclusion, we provide preclinical data in mice and NHPs for the application of adenine base editing to treat genetic liver diseases. We demonstrate therapeutically beneficial editing using transient and non-viral delivery vectors without induction of considerable off-target mutations.

The remaining risks of such clinically viable base editor therapies must be carefully weighed against their benefits. This makes genetic liver diseases, which at present can be cured only by organ transplantation, ideal candidates.

\section{Online content}

Any methods, additional references, Nature Research reporting summaries, source data, extended data, supplementary information, acknowledgements, peer review information; details of author contributions and competing interests; and statements of data and code availability are available at https://doi.org/10.1038/ s41587-021-00933-4.

Received: 5 February 2021; Accepted: 23 April 2021; Published online: 19 May 2021

\section{References}

1. Jinek, M. et al. A programmable dual-RNA-guided DNA endonuclease in adaptive bacterial immunity. Science 337, 816-821 (2012).

2. Hsu, P. D., Lander, E. S. \& Zhang, F. Development and applications of CRISPR-Cas9 for genome engineering. Cell https://doi.org/10.1016/j. cell.2014.05.010 (2014).

3. Mao, Z., Bozzella, M., Seluanov, A. \& Gorbunova, V. Comparison of nonhomologous end joining and homologous recombination in human cells. DNA Repair https://doi.org/10.1016/j.dnarep.2008.06.018 (2008).

4. van den Bosch, M., Lohman, P. H. M. \& Pastink, A. DNA double-strand break repair by homologous recombination. Biol. Chem. https://doi. org/10.1515/BC.2002.095 (2002).

5. Liu, M. et al. Methodologies for improving HDR efficiency. Front. Genet. https://doi.org/10.3389/fgene.2018.00691 (2019).

6. Zhao, X et al. Cell cycle-dependent control of homologous recombination. Acta Biochim Biophys Sin. https://doi.org/10.1093/abbs/gmx055 (2017).

7. Komor, A. C., Kim, Y. B., Packer, M. S., Zuris, J. A. \& Liu, D. R. Programmable editing of a target base in genomic DNA without double-stranded DNA cleavage. Nature 533, 420-424 (2016).

8. Gaudelli, N. M. et al. Programmable base editing of $\mathrm{A} \bullet \mathrm{T}$ to $\mathrm{G} \bullet \mathrm{C}$ in genomic DNA without DNA cleavage. Nature 551, 464-471 (2017).

9. Yeh, W.-H. et al. In vivo base editing restores sensory transduction and transiently improves auditory function in a mouse model of recessive deafness. Sci. Transl. Med. 12, eaay9101 (2020).

10. Yeh, W. H., Chiang, H., Rees, H. A., Edge, A. S. B. \& Liu, D. R. In vivo base editing of post-mitotic sensory cells. Nat. Commun. 9, 2184 (2018).

11. Fu, Y. et al. High-frequency off-target mutagenesis induced by CRISPR-Cas nucleases in human cells. Nat. Biotechnol. https://doi.org/10.1038/nbt.2623 (2013).

12. Hsu, P. D. et al. DNA targeting specificity of RNA-guided Cas9 nucleases. Nat. Biotechnol. https://doi.org/10.1038/nbt.2647 (2013).

13. Grünewald, J. et al. Transcriptome-wide off-target RNA editing induced by CRISPR-guided DNA base editors. Nature https://doi.org/10.1038/s41586019-1161-z (2019).

14. Grünewald, J. et al. CRISPR DNA base editors with reduced RNA off-target and self-editing activities. Nat. Biotechnol. https://doi.org/10.1038/s41587-0190236-6 (2019).

15. Zhou, C. et al. Off-target RNA mutation induced by DNA base editing and its elimination by mutagenesis. Nature https://doi.org/10.1038/ s41586-019-1314-0 (2019).

16. Villiger, L. et al. In vivo cytidine base editing of hepatocytes without detectable off-target mutations in RNA and DNA. Nat. Biomed. Eng. 5, 179-189 (2020).

17. Doman, J. L., Raguram, A., Newby, G. A. \& Liu, D. R. Evaluation and minimization of Cas9-independent off-target DNA editing by cytosine base editors. Nat. Biotechnol. 38, 620-628 (2020).

18. Gaudelli, N. M. et al. Directed evolution of adenine base editors with increased activity and therapeutic application. Nat. Biotechnol. 38, 892-900 (2020).

19. Park, S. W., Moon, Y. A. \& Horton, J. D. Post-transcriptional regulation of low density lipoprotein receptor protein by proprotein convertase subtilisin/ kexin type 9a in mouse liver. J. Biol. Chem. https://doi.org/10.1074/jbc. M410077200 (2004)

20. Stein, E. A. et al. Effect of a monoclonal antibody to PCSK9, REGN727/ SAR236553, to reduce low-density lipoprotein cholesterol in patients with heterozygous familial hypercholesterolaemia on stable statin dose with or without ezetimibe therapy: a phase 2 randomised controlled trial. Lancet https://doi.org/10.1016/S0140-6736(12)60771-5 (2012).

21. Carreras, A. et al. In vivo genome and base editing of a human PCSK9 knock-in hypercholesterolemic mouse model. BMC Biol. https://doi. org/10.1186/s12915-018-0624-2 (2019).

22. Chadwick, A. C., Wang, X. \& Musunuru, K. In vivo base editing of PCSK9 (proprotein convertase subtilisin/kexin type 9) as a therapeutic alternative to genome editing. Arterioscler. Thromb. Vasc. Biol. https://doi.org/10.1161/ ATVBAHA.117.309881 (2017).

23. Winter, J. et al. Targeted exon skipping with AAV-mediated split adenine base editors. Cell Discov. https://doi.org/10.1038/s41421-019-0109-7 (2019).

24. Kluesner, M. G. et al. CRISPR-Cas 9 cytidine and adenosine base editing of splice-sites mediates highly-efficient 3 disruption of proteins in primary cells. Preprint at bioRxiv https://doi.org/10.1101/2020.04.16.045336 (2020).

25. Anzalone, A. V., Koblan, L. W. \& Liu, D. R. Genome editing with CRISPRCas nucleases, base editors, transposases and prime editors. Nat. Biotechnol. https://doi.org/10.1038/s41587-020-0561-9 (2020).

26. Truong, D. J. J. et al. Development of an intein-mediated split-Cas 9 system for gene therapy. Nucleic Acids Res. 43, 6450-6458 (2015).

27. Richter, M. F. et al. Phage-assisted evolution of an adenine base editor with improved Cas domain compatibility and activity. Nat. Biotechnol. 38, 883-891 (2020) 
28. Duan, D. et al. Circular intermediates of recombinant adeno-associated virus have defined structural characteristics responsible for long-term episomal persistence in muscle tissue. J. Virol. https://doi.org/10.1128/jvi.72.11.85688577.1998 (1998).

29. Nelson, C. E. et al. Long-term evaluation of AAV-CRISPR genome editing for Duchenne muscular dystrophy. Nat. Med. 25, 427-432 (2019).

30. Finn, J. D. et al. A single administration of CRISPR/Cas9 lipid nanoparticles achieves robust and persistent in vivo genome editing. Cell Rep. 22, 2227-2235 (2018)

31. Yin, H. et al. Structure-guided chemical modification of guide RNA enables potent non-viral in vivo genome editing. Nat. Biotechnol. 35, 1179-1187 (2017).

32. Blouin, A., Bolender, R. P. \& Weibel, E. R. Distribution of organelles and membranes between hepatocytes and nonhepatocytes in the rat liver parenchyma. A stereological study. J. Cell Biol. https://doi.org/10.1083/ jcb.72.2.441 (1977).

33. Rees, H. A., Wilson, C., Doman, J. L. \& Liu, D. R. Analysis and minimization of cellular RNA editing by DNA adenine base editors. Sci. Adv. https://doi. org/10.1126/sciadv.aax5717 (2019).

34. McGrath, E. et al. Targeting specificity of APOBEC-based cytosine base editor in human iPSCs determined by whole genome sequencing. Nat. Commun. 10, 5353 (2019).

35. Zuo, E. et al. Cytosine base editor generates substantial off-target single-nucleotide variants in mouse embryos. Science 364, 289-292 (2019).

36. Jin, $\mathrm{S}$. et al. Cytosine, but not adenine, base editors induce genome-wide off-target mutations in rice. Science 364, 292-295 (2019).

37. Hou, Y. et al. Single-cell exome sequencing and monoclonal evolution of a JAK2-negative myeloproliferative neoplasm. Cell https://doi.org/10.1016/j. cell.2012.02.028 (2012)

38. Katsuda, T. et al. Conversion of terminally committed hepatocytes to culturable bipotent progenitor cells with regenerative capacity. Cell Stem Cell https://doi.org/10.1016/j.stem.2016.10.007 (2017).

39. Marquart, K. F. et al. Predicting base editing outcomes with an attention-based deep learning algorithm trained on high-throughput target library screens. Preprint at bioRxiv https://doi.org/10.1101/2020.07.05.186544 (2020)

40. Tsai, S. Q. et al. CIRCLE-seq: a highly sensitive in vitro screen for genome-wide CRISPR-Cas9 nuclease off-targets. Nat. Methods 14, 607-614 (2017).

41. Lazzarotto, C. R. et al. CHANGE-seq reveals genetic and epigenetic effects on CRISPR-Cas9 genome-wide activity. Nat. Biotechnol. https://doi.org/10.1038/ s41587-020-0555-7 (2020)
42. Katz, S.-F. et al. Disruption of Trp53 in livers of mice induces formation of carcinomas with bilineal differentiation. Gastroenterolgy https://doi. org/10.1053/j.gastro.2012.02.009 (2012).

43. Chen, Y.-W. et al. Loss of p53 and Ink4a/Arf cooperate in a cell autonomous fashion to induce metastasis of hepatocellular carcinoma cells. Cancer Res. https://doi.org/10.1158/0008-5472.CAN-07-0381 (2007).

44. Chahal, J. S. et al. An RNA nanoparticle vaccine against Zika virus elicits antibody and $\mathrm{CD}^{+} \mathrm{T}$ cell responses in a mouse model. Sci. Rep. https://doi. org/10.1038/s41598-017-00193-w (2017).

45. Liang, F. et al. Efficient targeting and activation of antigen-presenting cells in vivo after modified mRNA vaccine administration in rhesus macaques. Mol. Ther. https://doi.org/10.1016/j.ymthe.2017.08.006 (2017).

46. Nobles, C. L. et al. IGUIDE: an improved pipeline for analyzing CRISPR cleavage specificity. Genome Biol. https://doi.org/10.1186/s13059-019-1625-3 (2019).

47. Arbab, M. et al. Determinants of base editing outcomes from target library analysis and machine learning. Cell 182, 463-480 (2020).

48. Okano, Y. et al. Molecular basis of phenotypic heterogeneity in phenylketonuria. N. Engl. J. Med. 324, 1232-1238 (1991).

49. Kvittingen, E. A., Rootwelt, H., Berger, R. \& Brandtzaeg, P. Self-induced correction of the genetic defect in tyrosinemia type I. J. Clin. Invest. https://doi.org/10.1172/JCI117509 (1994).

50. Meijer, A. J., Lamers, W. H. \& Chamuleau, R. A. Nitrogen metabolism and ornithine cycle function. Physiol. Rev. https://doi.org/10.1152/ physrev.1990.70.3.701 (1990).

51. Blokzijl, F. et al. Tissue-specific mutation accumulation in human adult stem cells during life. Nature https://doi.org/10.1038/nature19768 (2016).

Publisher's note Springer Nature remains neutral with regard to jurisdictional claims in published maps and institutional affiliations.

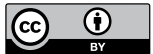

Open Access This article is licensed under a Creative Commons

Attribution 4.0 International License, which permits use, sharing, adaptation, distribution and reproduction in any medium or format, as long as you give appropriate credit to the original author(s) and the source, provide a link to the Creative Commons license, and indicate if changes were made. The images or other third party material in this article are included in the article's Creative Commons license, unless indicated otherwise in a credit line to the material. If material is not included in the article's Creative Commons license and your intended use is not permitted by statutory regulation or exceeds the permitted use, you will need to obtain permission directly from the copyright holder. To view a copy of this license, visit http://creativecommons. org/licenses/by/4.0/.

(c) The Author(s) 2021 


\section{Methods}

Cell culture, transfection and harvest. Hepa1-6 (ATCC CRL-18.30) and HEK293T (ATCC CRL-321) cells were maintained in DMEM plus GlutaMAX (Thermo Fisher Scientific), and HepG2 (ATCC HB-8065) cells were maintained in EMEM (Gibco). The media were supplemented with $10 \%$ (vol/vol) FBS and $1 \times$ penicillin-streptomycin (Thermo Fisher Scientific). Cells were maintained at $37^{\circ} \mathrm{C}$ and $5 \% \mathrm{CO}_{2}$ at a confluency below $90 \%$ and seeded on 48 -well cell culture plates (Greiner) for transfection. Then, $12-16 \mathrm{~h}$ after seeding, at approximately $70 \%$ confluency, cells were transfected using $1.5 \mu \mathrm{l}$ of Lipofectamine 2000 (Thermo Fisher Scientific) with $750 \mathrm{ng}$ of base editor plasmid (Addgene no. 112101) and 250 ng of sgRNA plasmid (Addgene no. 52963). HepG2 cells were transfected using the Neon Transfection System (Invitrogen) following the manufacturer's instructions. Briefly, $1.2 \times 10^{6}$ cells and $2.4 \mu \mathrm{g}$ of the ABEmax plasmid DNA (MHp27) and $0.9 \mu \mathrm{g}$ of the hPCSK9-gRNA (gRNA-6) plasmid DNA were resuspended in electroporation buffer R (Invitrogen) and electroporated with the following program: $1,230 \mathrm{~V}, 20 \mathrm{~ms}$ and three pulses. Transfection efficiency was checked by microscopy of GFP-positive cells and enriched by puromycin selection $\left(2.5 \mu \mathrm{g} \mathrm{ml}^{-1}\right)$. Cells were expanded until they reached confluency in a six-well plate. Upon detachment using TrypLE Express Enzyme (Thermo Fisher Scientific) for Hepa1-6 and HEK293T cells or Trypsin-EDTA for HepG2 cells, the cells were washed $2 \times$ in PBS and distributed for DNA lysis, RNA isolation or protein harvest.

Genomic DNA amplification, Sanger sequencing and BEAT analysis. Next, $30 \mu$ of a cell suspension in PBS was directly lysed using $10 \mu \mathrm{l}$ of $4 \times$ lysis buffer $(10 \mathrm{mM}$ Tris- $\mathrm{HCl}$ at $\mathrm{pH} 8,2 \%$ Triton X-100, $1 \mathrm{mM}$ EDTA, $1 \%$ freshly added proteinase $\mathrm{K}$ ) and incubated at $60^{\circ} \mathrm{C}$ for $60 \mathrm{~min}$ and $95^{\circ} \mathrm{C}$ for $10 \mathrm{~min}$. Target sites were amplified by polymerase chain reaction (PCR) using GoTaq G2 Hot Start Green Master Mix (Promega) and the respective primer pair (Supplementary Table 5). Amplification products were purified using Agencourt AMPure XP beads (Beckman Coulter) and sequenced with the respective in-sequence primers (Supplementary Table 5) via the Sanger method. Editing efficiency was determined by BEAT analysis ${ }^{52}$.

RNA isolation and RT-qPCR. RNA isolation was performed using the RNeasy Kit (Qiagen). cDNA was reverse transcribed using the GoScript Reverse Transcriptase Kit (Promega). RT-PCR was performed using GoTaq qPCR Master Mix (Promega) with specific primers for mouse or human PCSK9 and mouse B2M or human $\beta$-actin as housekeeping genes (Supplementary Table 5 ) and analyzed by QuantStudio 5 Real-Time PCR System (Thermo Fisher Scientific) or 7900HT Fast Real-Time PCR System (Applied Biosystems). Fold changes were calculated using the $\Delta \Delta C T$ method.

Western blot. Harvested Hepa1-6 cells were lysed in RIPA buffer containing protease inhibitor and PhosSTOP phosphatase inhibitor (Sigma-Aldrich). Protein amount was determined with the Pierce BCA Protein Assay Kit (Thermo Fisher Scientific), and equal amounts of proteins were separated by SDS-PAGE electrophoresis followed by transfer to nitrocellulose membrane (Sigma-Aldrich). Membranes were incubated with goat anti-mouse-Pcsk9 (1:10,000, cat. no. AF3985-SP, R\&D Systems) and rabbit anti- $\beta$-actin (1:3,000, cat. no. 4970S, Cell Signaling) or rabbit anti-GAPDH (1:5,000, cat. no. 4970, Abcam). HRP- or IRDye-conjugated secondary antibodies (donkey anti-goat: LI-COR cat. no. 92632214; anti-rabbit: LI-COR cat. no. 926-68073; Cell Signaling cat. no. 7074; Promega cat. no. V8051) were used, and signal was revealed by enhanced chemiluminescence substrate (Thermo Fisher Scientific) or fluorescence using LI-COR.

ELISAs. Human and NHP PCSK9 levels were determined using the Human Proprotein Convertase 9/PCSK9 Quantikine ELISA Kit (R\&D Systems, cat. no. DPC900), and mouse Pcsk9 levels were determined by using the Mouse Proprotein Convertase 9/PCSK9 Quantikine ELISA Kit (R\&D Systems, cat. no. MPC900) according to the manufacturer's instructions. Anti-Cas9- or anti-TadA-specific antibodies were determined by an in-house set-up direct ELISA. In short, $10 \mathrm{ng}$ of Cas9 or TadA were immobilized on 96-well polystyrene MaxiSorp plates (Thermo Fisher Scientific, cat. no. 439454) diluted in 1× ELISA Coating Buffer (BioRad, cat. no. BUF030A) for $2 \mathrm{~h}$ at room temperature. After washing in $1 \times$ ELISA Wash Buffer (Bio-Rad, cat. no. BUF031C), the wells were blocked for $30 \mathrm{~min}$ in ELISA BSA blocking solution (Bio-Rad, cat. no. BUF032C). For Cas9 detection, mouse-anti-Cas9 mAB (7A9-3A3; clone no. 14697T, Cell Signaling, cat. no. 14697) was used as positive control and standard curve. Plasma samples were diluted 1:20,000 for mouse plasma and 1:2,000 for NHP serum in Tris-buffered saline with Tween (TBS-T) and incubated for $2 \mathrm{~h}$ at room temperature. Goat-anti-mouse (SouthernBiotech, cat. no. 1030-05) or mouse-anti-monkey (SouthernBiotech, cat. no. 4700-05) HRP-linked secondary antibodies were used to detected protein-binding antibodies and developed using 1-Step Turbo TMB-ELISA Substrate Solution (Thermo Fisher Scientific, cat. no. 34022) and stopped after 20 min with Stop Solution for TMB Substrates (Thermo Fisher Scientific, cat. no. N301). Absorbance was measured at $450 \mathrm{~nm}$ and background at $540 \mathrm{~nm}$; the latter was subtracted for quantification. For further background control, a 5\% BSA coating was analyzed simultaneously.

Protein production. $\mathrm{His}_{6}-\mathrm{MBP}$-tev-TadA-tadA ${ }^{*}$ was expressed overnight at $18^{\circ} \mathrm{C}$ in Escherichia coli Rosetta 2 (DE3) (Novagen) cells upon induction of T7 RNA polymerase with IPTG. Cells were resuspended and lysed in $20 \mathrm{mM}$ HEPES-KOH pH 7.5, $200 \mathrm{mM} \mathrm{KCl}, 10 \mathrm{mM}$ imidazole and supplemented with protease inhibitors, using a Maximator High Pressure Homogenizer Type HPL6. Clarified lysate was loaded on a 15-ml Ni-NTA Superflow column (Qiagen) and eluted with $20 \mathrm{mM}$ HEPES- $\mathrm{KOH}$ pH 7.5, $200 \mathrm{mM} \mathrm{KCl}, 200 \mathrm{mM}$ imidazole. In the second step, TadA is further purified using a gradient elution from a 10-ml HiTrap Heparin HP column (GE Healthcare) equilibrated in $20 \mathrm{mM}$ HEPES-KOH pH 7.5, $100 \mathrm{mM} \mathrm{KCl}, 1 \mathrm{mM}$ DTT. Protein containing fractions are pooled, and affinity tag is removed using TEV protease with incubation overnight at $4^{\circ} \mathrm{C}$. Uncleaved TadA is removed using reverse nickel-affinity chromatography step, and the untagged TadA flowthrough is applied to a Superdex $20016 / 600$ column (GE Healthcare) and eluted in $20 \mathrm{mM}$ HEPES-KOH pH 7.5, $200 \mathrm{mM} \mathrm{KCl}$. Purified fractions were concentrated, flash frozen in liquid nitrogen and stored at $-80^{\circ} \mathrm{C}$.

Cloning. The sequences of the AAV constructs used in this work were generated by using pLV302 and pLV312.3 (Addgene plasmid nos.119943 and 119944) where regions of interest were exchanged using NEBuilder HiFi DNA Assembly Master Mix (NEB no. E2621). Amino acid sequences are listed in Supplementary Note 1. PCR was performed using Q5 High-Fidelity DNA Polymerase (New England Biolabs).pCMV_ABEmax_P2A_GFP was a gift from David Liu (Addgene plasmid no. 112101). lentiGuide-Puro was a gift from Feng Zhang (Addgene plasmid no. 52963). The coding sequence of ABEmax was cloned into the mRNA production plasmid behind a T7 promoter for mRNA production and into pET His6 LIC cloning vector (2Bc-T, Addgene plasmid no. 37236) for protein production.

AAV vector production. All pseudotyped AAV8 vectors were produced by the Viral Vector Facility of the Neuroscience Center Zurich. AAV vectors were ultracentrifuged and diafiltered. Physical titers (vector genomes $\mathrm{ml}^{-1}$ ) were determined using a Qubit 3.0 Fluorometer. Identity of the packaged genomes of each AAV vector was confirmed by Sanger DNA sequencing.

Animal studies. Mouse experiments were performed in accordance with protocols approved by the Kantonales Veterinäramt Zürich. Mice were housed in a pathogen-free animal facility at the Institute of Molecular Health Sciences at ETH Zurich and kept in a temperature- and humidity-controlled room on a 12-h light/dark cycle. For long-term studies of mice with sensitized background, conditional Trp53 $3^{\mathrm{F} 2-10 / \mathrm{F2}-10}$ knockout mice ${ }^{53}$ were mated with albumin $(\mathrm{Alb})$-Cre transgenic mice ${ }^{54}$. Mice were fasted for 3-4h before blood was collected from the inferior vena cava before liver perfusion. Mice were injected with $1-3 \mathrm{mg} \mathrm{kg}^{-1}$ of total RNA (LNP) or $1 \times 10^{12}$ AAV vector genomes per mouse at 5 weeks of age. Injection volumes were $120-150 \mu \mathrm{l}$. Only male C57BL/6J animals were used. Male and female Alb-Cre $\times$ Trp5 $3^{\text {flox/llox }}$ animals were used (untreated group: 17 males and 12 females; AAV-only group: 16 males and two females; AAV-treated group: 16 males and nine females). Studies involving NHPs were conducted at a facility accredited by the Association for Assessment and Accreditation of Laboratory Animal Care International, operating in accordance with the principles of the U.S. Food and Drug Administration's Good Laboratory Practice and the Guide for the Care and Use of Laboratory Animals from the Institute of Laboratory Animal Resources (2011). All protocols were reviewed and approved by the Acuitas animal care and use committee. Male $M$. fascicularis (approximately 2 years of age) were housed in a temperature- and humidity-controlled room on a 12-h light/dark cycle. Animals received a 60 -min intravenous infusion of $0.75 \mathrm{mg} \mathrm{kg}^{-1}$ or $1.5 \mathrm{mg} \mathrm{kg}^{-1}$ of total RNA, formulated in LNP and diluted in $0.9 \%$ sodium chloride USP. A volume of $5 \mathrm{ml} \mathrm{kg} \mathrm{kg}^{-1}$ was administered by a $1-\mathrm{h}$ infusion via the cephalic vein. Animals were fasted for $4 \mathrm{~h}$ before serum collection for ELISA and clinical chemistry.

RNA synthesis and LNP encapsulation. Heavily modified sgRNA (P1) for mouse studies was synthesized using Synthego's CRISPRevolution platform using solid-phase phosphoramidite chemistry. After synthesis and a series of post-processing steps and purification, oligonucleotides were quantified by ultraviolet (UV) absorption, and their identity and quality were confirmed using an Agilent 1290 Infinity II liquid chromatography system coupled with an Agilent 6530B Quadrupole time-of-flight mass spectrometry (Agilent Technologies) in a negative ion polarity mode. Chemically ultra-heavily modified sgRNAs (P2) were ordered from Agilent Technologies. Heavily modified sgRNAs for large-scale production for NHP studies were synthesized using an ÄKTA Oligopilot Plus 100 oligonucleotide synthesizer at a $112-\mu \mathrm{mol}$ scale. After synthesis and de-protection steps, the oligo was subjected to solid-phase extraction using an ÄKTA Explorer FPLC system. This material then underwent further purification and quality assessment using the Agilent 1200 HPLC System. HPLC fractions were selected, combined and processed by tangential flow filtration using the Pall Minimate EVO TFF system. Final product quantity was evaluated using UV absorption, and its identity and quality were confirmed using Agilent 1290 Infinity II liquid chromatography system coupled with an Agilent 6530B Quadrupole time-of-flight mass spectrometry (Agilent Technologies) in a negative ion polarity mode.

The coding sequence of ABEmax was cloned into the mRNA production plasmid. mRNA production and LNP encapsulation were performed as described ${ }^{55}$. Briefly, mRNAs were transcribed to contain 101 nucleotide-long poly(A) tails. m1 $\Psi$-5' -triphosphate (TriLink no. N-1081) instead of UTP was used to generate 
modified nucleoside-containing mRNA. Capping of the in vitro transcribed mRNAs was performed co-transcriptionally using the trinucleotide cap1 analog, CleanCap (TriLink, no. N-7413). mRNA was purified by cellulose (Sigma-Aldrich, no. 11363-250G) purification as described ${ }^{56}$. All mRNAs were analyzed by agarose gel electrophoresis and were stored frozen at $-20^{\circ} \mathrm{C}$. Cellulose-purified $\mathrm{m} 1 \Psi$-containing mRNA, together with the synthesized sgRNA, were encapsulated in LNPs. LNPs were formulated as described previously $y^{57}$. In short, an ethanolic solution of 1,2-distearoyl-sn-glycero-3-phosphocholine, cholesterol, a PEG lipid and an ionizable cationic lipid was rapidly mixed with an aqueous solution ( $\mathrm{pH} 4)$ containing SpCas9-ABEmax mRNA and sgRNA (1:1 weight ratio) using an in-line mixer. The ionizable lipid and LNP composition are described in U.S. patent application US 2016/0376224 A1 (2016), with the ionizable lipid (pKa in the 6.0-6.5 range) belonging to lipid class defined by the structure shown in Supplementary Fig. 4a. The resulting LNP formulation was dialyzed overnight against $1 \times \mathrm{PBS}, 0.2-\mu \mathrm{m}$ sterile filtered and stored at $-80^{\circ} \mathrm{C}$ at a concentration of $1 \mu \mathrm{g} \mu \mathrm{l}^{-1}$ of total RNA. LNP had an average hydrodynamic diameter of $67-71 \mathrm{~nm}$ with a polydispersity index of $0.02-0.06$ as determined by dynamic light scattering (Malvern Nano ZS Zetasizer) and a mode size of $67-75 \mathrm{~nm}$ as determined by nanoparticle tracking analysis (Malvern Panalytical NanoSight NS300). Encapsulation efficiencies of SpCas9-ABEmax mRNA and sgRNA in the LNP were both at $96 \%$ measured by the Quant-iT Ribogreen Assay (Life Technologies). Acuitas will provide the LNP used in this work to any academic investigator who would like to test it.

Primary hepatocyte isolation. Mice were euthanized using $\mathrm{CO}_{2}$ and immediately perfused with Hank's balanced salt solution (Thermo Fisher Scientific) plus $0.5 \mathrm{mM}$ EDTA via the inferior vena cava and a subsequent incision in the portal vein. During this step, one liver lobe was squeezed off via a thread to inhibit perfusion of this lobe to collect whole liver samples for embedding and whole liver lysates. After blanching of the liver, mice were perfused with digestion medium (low-glucose DMEM plus $1 \times$ penicillin-streptomycin (Thermo Fisher Scientific), $15 \mathrm{mM}$ HEPES and freshly added Liberase (Roche)) for $5 \mathrm{~min}$. Livers were isolated in cold isolation medium (low-glucose DMEM supplemented with 10\% (vol/vol) FBS plus $1 \times$ penicillin-streptomycin (Thermo Fisher Scientific) and GlutaMax (Thermo Fisher Scientific)), and the liver was gently dissociated to yield a cell suspension that was passed through a $100-\mu \mathrm{m}$ filter. The suspension was then centrifuged at $50 \mathrm{~g}$ for $2 \mathrm{~min}$ and washed with isolation medium 2-3 times until the supernatant was clear. The primary hepatocytes were pelleted for DNA or RNA isolation.

Genomic DNA isolation and HTS. Genomic DNA from mouse tissues was isolated using the DNeasy Blood and Tissue Kit (Qiagen) according to the manufacturer's protocol or directly lysed using direct lysis buffer: $10 \mu \mathrm{l}$ of $4 \times$ lysis buffer (10 mM Tris-HCl pH 8, 2\% Triton X-100, 1 mM EDTA, 1\% freshly added proteinase $\mathrm{K}$ ) and incubated at $60^{\circ} \mathrm{C}$ for $60 \mathrm{~min}$ and $95^{\circ} \mathrm{C}$ for $10 \mathrm{~min}$. Target sites were amplified by PCR using GoTaq G2 Hot Start Green Master Mix (Promega) or NEBNext High-Fidelity $2 \times$ PCR Master Mix and the respective primer pair (Supplementary Table 6 ) in 26 cycles. The PCR product was purified using $0.8 \times$ Agencourt AMPure XP beads (Beckman Coulter) and amplified with primers containing sequencing adaptors for another six cycles. The products were gel purified and quantified using the Qubit 3.0 fluorometer with the dsDNA HS Assay Kit (Thermo Fisher Scientific). Samples were sequenced on Illumina MiSeq. After demultiplexing, the samples were analyzed using CRISPResso2 (ref. ${ }^{58}$ ).

Clinical chemistry and cyokine and inflammatory biomarkers. Total cholesterol, triglyceride, high-density lipoprotein (HDL), AST and ALT levels from all mouse samples were measured as routine parameters at the Division of Clinical Chemistry and Biochemistry at the University Children's Hospital Zurich using Alinity ci-series. LDL levels were calculated by using the Friedewald formula. NHP serum was subjected to a full clinical chemistry panel, including ALT, AST, total bilirubin, LDL cholesterol, HDL cholesterol and total cholesterol. Approximately $1 \mathrm{ml}$ of blood was taken from the femoral vein, processed to serum and analyzed using a Beckman Coulter analyzer. For cytokine and inflammatory biomarker analyses, approximately $0.8 \mathrm{ml}$ of blood was processed to serum, and a panel of ten cytokine and inflammatory biomarkers (IFN- $\alpha 2 a$, IL-18, IL-1RA, IL-1 $\beta$, IL-6, IP-10, MCP-1, MIP-1 $\alpha$, MIP- $1 \beta$ and TNF- $\alpha$ ) was evaluated using U-PLEX Biomarker Group 1 (NHP) assay kits (Meso Scale Diagnostics).

Tissue cryosections. Mouse livers were perfused with Hank's buffer and bound off before further perfusion. The separated section was fixed in $4 \%$ paraformaldehyde (PFA) at $4^{\circ} \mathrm{C}$ overnight. Tissues were transferred to a $30 \%$ sucrose solution overnight at $4^{\circ} \mathrm{C}$ and embedded in OCT compound in cryomolds (Tissue-Tek). Frozen tissues were sectioned at $7 \mu \mathrm{m}$ at $-20^{\circ} \mathrm{C}$, and mounted directly on Superfrost Plus slides (Thermo Fisher Scientific). Cryosections were counterstained with DAPI (Thermo Fisher Scientific) and mounted in VECTASHIELD mounting medium (Vector Labs). Two frozen sections were analyzed per mouse per tissue.

Single-molecule fluorescence in situ hybridization. The ABEmax probe library was designed using Stellaris FISH Probe Designer Software (Biosearch Technologies) (Supplementary Table 7) and coupled to Cy5 as described ${ }^{9}$. Livers were fixed in $4 \%$ PFA in PBS for $3 \mathrm{~h}$ and subsequently agitated in $30 \%$ sucrose and $4 \%$ PFA in PBS overnight at $4{ }^{\circ} \mathrm{C}$. Fixed tissues were embedded in Tissue-Tek OCT Compound (Sakura, 4583). Then 8 - $\mu$ m-thick sections were sectioned onto poly-L-lysine-coated coverslips, air dried for $5 \mathrm{~min}$, fixed for $15 \mathrm{~min}$ in $4 \% \mathrm{PFA}$ and permeabilized overnight in $70 \% \mathrm{EtOH}$. The liver sections were hybridized with single-molecule fluorescence in situ hybridization (smFISH) probe sets according to a previously published protocol ${ }^{60}$. DAPI (Sigma-Aldrich) was used as nuclear counterstain. smFISH imaging was performed on a Leica THUNDER 3D live cell imaging system, using the following THUNDER computational clearing settings: Feature Scale (nm): 350; Strength (\%): 98; Deconvolution settings: Auto; and Optimization: High.

Histology and staining. Tissues were fixed using $4 \%$ PFA at $4{ }^{\circ} \mathrm{C}$ overnight and dehydrated the next day before paraffinization. Paraffin blocks were cut into 5 - $\mu \mathrm{m}$-thick sections, deparaffinized with xylene and rehydrated. Sections were stained for hematoxylin and eosin (H\&E) or Sirius Red and examined for histopathological changes.

Microscopy. Mouse tissue was imaged using a Zeiss Apotome. Imaging conditions and intensity scales were matched for all images. Images were taken using Zeiss software Zen 2 and analyzed by Fiji ImageJ software (v1.51n $)^{61}$

Guide-dependent off-target prediction and analysis. For CIRCLE-seq and CHANGE-seq, the sgRNA was first tested for functionality by digesting the Sanger amplicon described above. The library was prepared as previously described ${ }^{40,41}$. Data were processed using version 1.1 of the CIRCLE-seq analysis pipeline (https:// github.com/tsailabSJ/circleseq) with the following parameters: 'window_size: 3; mapq_threshold: 50; start_threshold: 1 ; gap_threshold: 3 ; mismatch_threshold: 6; merged_analysis: True, variant_analysis: True. The respective target sites were deep sequenced and covered by at least 10,000 reads per site. Highly repetitive sequences were further processed by extracting the amplicon with cutadapt ${ }^{62}$ (v3.1) excluding the protospacer region. If this was also not possible because the region was too similar to a different site in the genome, the off-target editing events could not be determined. For iGUIDE, libraries were prepared as previously described ${ }^{46}$.

RNA-seq experiments and data analysis. RNA library preparation was performed using the TruSeq Stranded Total RNA Kit (Illumina) with ribosomal RNA (rRNA) deletion. RNA-seq libraries were sequenced on an Illumina NovaSeq machine at the Functional Genomics Center Zurich, achieving an average of more than 160 million paired-end (PE) reads per sample. Quality control, pre-processing, alignment of RNA-seq reads: Quality of Illumina PE RNA-seq reads was evaluated using FastQC version 0.11 .7 (https://www.bioinformatics.babraham.ac.uk/projects/ fastqc/). Using FastQ Screen version 0.11.1 (https://www.bioinformatics.babraham. ac.uk/projects/fastq_screen/), potential sample contaminations (genomic DNA, rRNA and mycoplasma) were screened against a custom database including UniVec (https://www.ncbi.nlm.nih.gov/tools/vecscreen/univec/), RefSeq mRNA sequences, selected genome sequences (human, mouse, arabidopsis, bacteria, virus, phix, lambda and mycoplasma) (https://www.ncbi.nlm.nih.gov/refseq/) and SILVA rRNA sequences (https://www.arb-silva.de/). Illumina PE reads were pre-processed using fastp version 0.20 .0 to trim off sequencing adaptors and low-quality ends (average quality lower than 20 within a 4 -nt window). Flexbar version 3.0.3 was used to remove the first six bases of each read, which showed priming bias introduced by the library preparation protocol ${ }^{63}$. PE reads longer than $50 \mathrm{bp}$ were trimmed to $50 \mathrm{bp}$ before being aligned to remove overlapping reads ends, which can inflate allele frequency calculation and variant calls. Quality controlled reads (average quality 20 and above, read length 20 and above) were aligned to the reference genomes (mouse reference genome: GRCm38.p5, Ensembl release 91; human reference genome: GRCh38.p10, Ensembl release 91) using STAR version 2.7.0e with two-passes mode. PCR duplicates were marked using Picard version 2.9.0. Read alignments were comprehensively evaluated in terms of different aspects of RNA-seq experiments, such as sequence quality, genomic DNA and rRNA contamination, GC/PCR/sequence bias, sequencing depth, strand specificity, coverage uniformity and read distribution over the genome annotation, using R scripts in ezRun (https://github.com/uzh/ezRun/) developed at the Functional Genomics Center Zurich. RNA sequence variant calling and filtering: Variant calling from RNA-seq reads was performed according to Genome Analysis Toolkit (GATK) Best Practices (https://gatkforums.broadinstitute.org/ gatk/discussion/3891/calling-variants-in-rnaseq). In detail, GATK (v4.1.2.0) tool SplitNCigarReads was applied to post-process the read alignments. Afterwards, variants were called using HaplotypeCaller (GATK v4.1.2.0) on PCR-deduplicated, post-processed aligned reads. Variant loci in base editor overexpression experiments were filtered to exclude sites without high-confidence reference genotype calls in the control experiment. For a given SNV, the read coverage in the control experiment should be above the 90th percentile of the read coverage across all SNVs in the corresponding overexpression experiment. Only loci having at least $99 \%$ of reads containing the reference allele in the control experiment were kept. Only sites with at least ten reads in the treated sample were considered. Quantification of gene expression: Transcript expression was calculated using kallisto (v0.44.0). 
WGS and data analysis. Upon confirmation of on-target editing, DNA was harvested using the QIAamp DNA Mini Kit (Qiagen) or Quick DNA Microprep Kit (Zymo Research) according to manufacturer instructions. DNA concentrations were determined using the Qubit dsDNA HS Kit (Invitrogen). WGS was performed at a mean coverage of $30 \times$ using an Illumina NovaSeq. Read alignment, variant calling and variant filtering: Sequence reads were mapped against mouse reference genome GRCm 38 by using the Burrows-Wheeler Aligner version 0.7.5 mapping tool ${ }^{64}$ with settings 'bwa mem -c 100 -M'. Sequence reads were marked for duplicates by using Sambamba version 0.4.732 and realigned per donor by using the GATK IndelRealigner version 2.7.2. Raw variants were multisample-called by using the GATK HaplotypeCaller version 3.4-46 (ref. ${ }^{65}$ ) and GATK-Queue version 3.4-46 with default settings and additional option 'EMIT_ALL CONFIDENT SITES. The quality of variant and reference positions was evaluated by using GATK VariantFiltration version 3.4-46 with options '-snpFilterName LowQualityDepth -snpFilterExpression “QD $<2.0$ ” -snpFilterName MappingQuality -snpFilterExpression "MQ $<40.0$ ” -snpFilterName StrandBias -snpFilterExpression "FS $>60.0$ " -snpFilterName HaplotypeScoreHigh -snpFilterExpression "HaplotypeScore > 13.0" -snpFilterName MQRankSumLow -snpFilterExpression "MQRankSum <-12.5” -snpFilterName ReadPosRankSumLow -snpFilterExpression "ReadPosRankSum $<-8.0$ " -cluster 3 -window 35'. Full pipeline description and settings are also available at https:// github.com/UMCUGenetics/IAP. To obtain high-quality somatic mutation catalogs, we applied post-processing filters as described ${ }^{51}$. Briefly, we considered variants at autosomal chromosomes without any evidence from a paired control sample (genomic DNA isolated from untreated tissue from the same mouse); passed by VariantFiltration with a GATK phred-scaled quality score $\geq 100$ for base substitutions and $\geq 250$ for indels; a base coverage of at least $20 \times$ in the clonal and paired control sample; mapping quality $\geq 60$; and no overlap with single-nucleotide polymorphisms in the Single Nucleotide Polymorphism Database version 142. We additionally filtered base substitutions with a GATK genotype quality (GQ) score lower than 99 or 10 in clonal or paired control samples, respectively. For indels, we filtered variants with a GQ score lower than 99 in both clonal and paired control samples and filtered indels that were present within $100 \mathrm{bp}$ of a called variant in the control sample. In addition, for both SNVs and indels, we considered only variants with a variant allele frequency (VAF) of 0.2 or higher in the clones to exclude in vitro accumulated mutations ${ }^{51,66}$. The scripts are available at https://github.com/ToolsVanBox/SNVFI and https://github.com/ ToolsVanBox/INDELFI. Owing to the karyotypically unstable nature of the cells and for the fair comparison of the number of mutations in the later analysis, only the mutations from the regions considered as diploid $(1.5<$ ratio $<2.5$ from the Control-FREEC ${ }^{67}$ output when the samples were treated as diploid) and callable were included. The absolute number of mutations was corrected for the lengths of the accounted genomic regions. Mutational profile and signature analysis: The numbers of six substitution types $(C>A, C>G, C>T, T>A, T>C$ and $T>G)$ or 96-trinucleotide mutation types (six substitution types with $5^{\prime}$ and $3^{\prime}$ flanking bases) were reported, and the frequencies of the 96-trinucleotide mutations were plotted for every mouse using an in-house-developed R package ${ }^{68}$. For the normalized absolute number and relative amount of six substitution types, the samples were classified based on the injected chemicals; for each group, the mean and standard deviation were calculated and plotted. To illustrate the potential TadA activity in the samples, the identified TadA motif ${ }^{39}$ was used as TadA signature for cosine similarity comparison. The 96-trinuclueotide frequencies were pooled from the two signatures and normalized so that the frequencies add up to 1 . The 96-nt TadA signature was deduced under the assumption that other substitutions do not contribute to the TadA signature. For the three control mouse samples, the 96-nt mutational profile was constructed and normalized by the total number of SNVs and multiplied by the median number of SNVs (428 SNVs) to make them comparable between the samples. To mimic the TadA activity on the mutational profile, the additional number of SNVs were distributed over the 96-nt mutational patterns according to the determined TadA signature, for 10, 25, 50 and 100 SNVs. Any decimal values were rounded and summed to the profiles of the controls. For all the samples and the TadA signature-added controls, cosine similarity with the TadA signature was calculated using MutationalPatterns ${ }^{68}$ in R. To calculate the variant detection sensitivity of our method, we identified germline variants and counted how many of them were found in the clones. To exclude potential artifacts in our data, the direct output from the IAP pipeline was further filtered with the following criteria: located in diploid and CALLABLE region, passed by VariantFiltration with a GATK phred-scaled quality score $\geq 100$, GATK GC score equals 99 , base coverage of at least $20 \times$ in all the clones and the bulk samples, does not overlap with the variants in our blacklists (available upon reasonable request) and present as a heterozygous variant (VAF $\geq 0.3$ ) in the three bulk samples. Our filtering resulted in 86 heterozygous variants, and any position with VAF $<0.3$ was counted as absent in the clones. The global maximum-likelihood estimates and the confidence intervals for both mice groups were calculated using the $\mathrm{dNdScv}$ package ${ }^{69}$ and plotted using ggplot2 (ref. ${ }^{70}$ ) in R. Called SNVs were compared among groups using the online tool by the van de Peer lab (http://bioinformatics.psb.ugent.be/webtools/Venn/) provided by the VIB/ UGent. Comparison of more than six groups was analyzed and but retrospectively visualized using Adobe Illustrator.
Statistical analyses. A priori power calculations to determine sample sizes for animal experiments were performed using $\mathrm{G}^{\star} \mathrm{Power}^{71}$. Statistical analyses were performed using GraphPad Prism 8.0.0 for macOS. Sample sizes and the statistical tests used are described in the figure legends. $P<0.05$ was considered statistically significant.

Reporting Summary. Further information on research design is available in the Nature Research Reporting Summary linked to this article.

\section{Data availability}

The main data supporting the results in this study are available in the paper and its Supplementary Information. High-throughput sequencing data are publicly available under the following accession numbers: GSE168365 (Gene Expression Omnibus datasets for targeted amplicon sequencing and RNA-seq) and PRJEB41832 (Sequence Read Archive dataset for WGS samples).

\section{Code availability}

Scripts used to quantify on-target and off-target editing are listed in the Methods.

\section{References}

52. Xu, L., Liu, Y. \& Han, R. BEAT: a Python program to quantify base editing from Sanger sequencing. CRISPR J. https://doi.org/10.1089/crispr.2019.0017 (2019)

53. Jonkers, J. et al. Synergistic tumor suppressor activity of BRCA2 and p53 in a conditional mouse model for breast cancer. Nat. Genet. https://doi. org/10.1038/ng747 (2001).

54. Postic, C. \& Magnuson, M. A. DNA excision in liver by an albumin-Cre transgene occurs progressively with age. Genesis 26, 149-150 (2000).

55. Freyn, A. W. et al. A multi-targeting, nucleoside-modified mRNA influenza virus vaccine provides broad protection in mice. Mol. Ther. https://doi. org/10.1016/j.ymthe.2020.04.018 (2020).

56. Baiersdörfer, M. et al. A facile method for the removal of dsRNA contaminant from in vitro-transcribed mRNA. Mol. Ther. Nucleic Acids https://doi.org/10.1016/j.omtn.2019.02.018 (2019).

57. Conway, A. et al. Non-viral delivery of zinc finger nuclease mRNA enables highly efficient in vivo genome editing of multiple therapeutic gene targets. Mol. Ther. 27, 866-877 (2019).

58. Clement, K. et al. CRISPResso 2 provides accurate and rapid genome editing sequence analysis. Nat. Biotechnol. 37, 224-226 (2019).

59. Lyubimova, A. et al. Single-molecule mRNA detection and counting in mammalian tissue. Nat. Protoc. https://doi.org/10.1038/nprot.2013.109 (2013).

60. Itzkovitz, S. et al. Single-molecule transcript counting of stem-cell markers in the mouse intestine. Nat. Cell Biol. https://doi.org/10.1038/ncb2384 (2012).

61. Schindelin, J. et al. Fiji: an open-source platform for biological-image analysis. Nat. Methods 9, 676-682 (2012).

62. Martin, M. Cutadapt removes adapter sequences from high-throughput sequencing reads. EMBnet.journal https://doi.org/10.14806/ej.17.1.200 (2011).

63. Hansen, K. D., Brenner, S. E. \& Dudoit, S. Biases in Illumina transcriptome sequencing caused by random hexamer priming. Nucleic Acids Res. 38, e131 (2010).

64. Li, H. Aligning sequence reads, clone sequences and assembly contigs with BWA-MEM. Preprint at https://arxiv.org/abs/1303.3997 (2013).

65. Depristo, M. A. et al. A framework for variation discovery and genotyping using next-generation DNA sequencing data. Nat. Genet. 43, 491-501 (2011).

66. Jager, M. et al. Measuring mutation accumulation in single human adult stem cells by whole-genome sequencing of organoid cultures. Nat. Protoc. 13, 59-78 (2018).

67. Boeva, V. et al. Control-FREEC: a tool for assessing copy number and allelic content using next-generation sequencing data. Bioinformatics $\mathbf{2 8}$, 423-425 (2012)

68. Blokzijl, F., Janssen, R., van Boxtel, R. \& Cuppen, E. MutationalPatterns: comprehensive genome-wide analysis of mutational processes. Genome Med. 10, 1-11 (2018)

69. Martincorena, I. et al. Universal patterns of selection in cancer and somatic tissues. Cell https://doi.org/10.1016/j.cell.2017.09.042 (2017).

70. Wickham, H. ggplot2: Elegant Graphics for Data Analysis (Springer, 2016); https://doi.org/10.1007/978-3-319-24277-4

71. Faul, F., Erdfelder, E., Lang, A. G. \& Buchner, A. GPower 3: a flexible statistical power analysis program for the social, behavioral, and biomedical sciences. Behav. Res. Methods https://doi.org/10.3758/BF03193146 (2007).

\section{Acknowledgements}

We thank the Functional Genomics Center Zurich for instrument use and, in particular, C. Aquino and M. D. Moccia for their guidance and support with respect to NGS and WGS; S. Iyer and J. K. Joung for guidance on variant filtering for transcriptome off-target identification; $\mathrm{Y}$. Li and S. Tsai for helpful discussions related to CHANGE-seq analysis; N. Rimann for support with blood sample analysis; and the Division of Clinical Chemistry and Biochemistry at the University Children's Hospital Zurich for analyzing 
routine parameters in mouse blood samples. We thank J. Huotari for comments on the manuscript. This work was supported by Swiss National Science Foundation grant no. 310030_185293 (to G.S.), Swiss National Science Foundation Sinergia grant no. 180257 (to B.T.), Swiss National Science Foundation grant no. 320030_176088 (to J.H.), Novartis Foundation for Medical-Biological Research grant no. 19A004 (to D. Witzigmann), PHRT grant no. 528 (to G.S., M.S., J.H. and B.T.), the Helmut Horten Foundation (to G.S.) and grants R01-HL134839, U19-AI142596, R01-AI124429 and HHS-NIH-NIAID-BAA2018 (to D. Weissman).

\section{Author contributions}

T.R. designed the study, performed experiments, analyzed data and wrote the manuscript. M.K.D. designed the study and analyzed data. P.J.C.L. developed, prepared and characterized lipid nanoparticles and contributed to the design of the study. R.O. and R.v.B. performed WGS analysis. Y.K.T., D. Witzigmann, M.S., B.T. and J.H. contributed to the design of the study. L.V., M.H., L.K., C.B., S.E., N.F., N.B. and M.P. performed experiments. W.Q. analyzed RNA-seq data. D.L. performed the pathological analysis for this study. N.P. and D. Weissman produced modified mRNAs. K.H., J.A.W., D.V.V. and A.P.K. performed the synthesis of modified sgRNAs. S.K. and Z.K. performed CIRCLE-seq, CHANGE-seq and iGUIDE. A.M. and M.J. provided conceptual advice and field-specific expertise. S.C.S. designed and supervised the study and wrote the manuscript. G.S. designed and supervised the study and wrote the manuscript. All authors approved the final version.

\section{Competing interests}

P.J.C.L., M.D., Y.K.T. and S.C.S. are employees of Acuitas Therapeutics. K.H., J.A.W., D.V.V. and A.P.K. are employees and shareholders of Synthego Corporation.

D. Weissman is named on patents that describe the use of nucleoside-modified mRNA as a platform to deliver therapeutic proteins. M.J., G.S. and L.V. are named on patents and patent applications related to CRISPR-Cas technologies. The remaining authors declare no competing financial interests.

\section{Additional information}

Supplementary information The online version contains supplementary material available at https://doi.org/10.1038/s41587-021-00933-4.

Correspondence and requests for materials should be addressed to S.C.S. or G.S.

Peer review information Nature Biotechnology thanks Markus Grompe and the other, anonymous, reviewer(s) for their contribution to the peer review of this work.

Reprints and permissions information is available at www.nature.com/reprints. 


\section{Reporting Summary}

Nature Research wishes to improve the reproducibility of the work that we publish. This form provides structure for consistency and transparency in reporting. For further information on Nature Research policies, see our Editorial Policies and the Editorial Policy Checklist.

\section{Statistics}

For all statistical analyses, confirm that the following items are present in the figure legend, table legend, main text, or Methods section.

$\mathrm{n} / \mathrm{a}$ Confirmed

$\bigotimes$ The exact sample size $(n)$ for each experimental group/condition, given as a discrete number and unit of measurement

A statement on whether measurements were taken from distinct samples or whether the same sample was measured repeatedly

The statistical test(s) used AND whether they are one- or two-sided

Only common tests should be described solely by name; describe more complex techniques in the Methods section.

$\triangle \square$ A description of all covariates tested

Х $\square$ A description of any assumptions or corrections, such as tests of normality and adjustment for multiple comparisons

A full description of the statistical parameters including central tendency (e.g. means) or other basic estimates (e.g. regression coefficient) AND variation (e.g. standard deviation) or associated estimates of uncertainty (e.g. confidence intervals)

For null hypothesis testing, the test statistic (e.g. $F, t, r$ ) with confidence intervals, effect sizes, degrees of freedom and $P$ value noted Give $P$ values as exact values whenever suitable.

Х $\square$ For Bayesian analysis, information on the choice of priors and Markov chain Monte Carlo settings

Х $\square$ For hierarchical and complex designs, identification of the appropriate level for tests and full reporting of outcomes

Х $\square$ Estimates of effect sizes (e.g. Cohen's $d$, Pearson's $r$ ), indicating how they were calculated

$$
\text { Our web collection on statistics for biologists contains articles on many of the points above. }
$$

\section{Software and code}

Policy information about availability of computer code

Data collection Next-generation sequencing (NGS) data was collected and demultiplexed by Illumina NovaSeq Control software (v1.7) and MiSeq Control software (v3.1.); Absorbance measurements were collected with BioTek Synegy HT; RT-qPCR data were collected using QuantStudio 5 RealTime PCR System (Thermo Fisher Scientific) or 7900HT Fast Real-Time PCR System (Applied Biosystems).

Data analysis NGS data were analyzed by CRISPResso2. Scrips for batch on- and off-target evaluation is added to the Supplement. PE reads were preprocessed using Fastp version 0.20.0; PCR-duplicates were marked using Picard version 2.9.0 and Read alignments were comprehensively evaluated using ezRun (https://github.com/uzh/ezRun/). STAR version 2.7.0e was used to map RNA-seq data. Variant calling was performed using GATK v4.1.2.0. using HaplotypeCaller and transcript expression was calculated using kallisto v0.44.0. WGS reads were aligned with BWA v0.7.5. mapping tool; marked for duplicated by using Sambamba v0.4.732 and realigned per donor by using GATK Indel Realigner v2.7.2. Raw variants were multisample-called by using the GATK HeplotypeCAller v3.4-46 and GATK-Queue v3.4-46 and compared to Nucleotide Polymorphism Database v142. The quality of variant and reference positions was evaluated by using GATK VariantFiltration v3.4-46. Diploidy assessment was performed using Control-FREEC v11.6. To exclude in vitro accumulated mutations we used SNVFI and inDelfi software: scripts are available at https://github.com/ToolsVanBox/SNVFI and https://github.com/ToolsVanBox/INDELFI. Called SNVs were compared between groups using the online tool by the van de Peer Lab http://bioinformatics.psb.ugent.be/webtools/Venn/ provided by the VIB/UGent. Cosine similarity with the TadA signature was calculated using MutationalPatterns in Rv4.0.3. The global maximum-likelihood estimates were calculated using dNdScv package v.0.1.0 and plotted using ggplot2 in R v4.0.3. Additional data visualization was performed using Adobe Illustrator v22.1. Images were taken using Zeiss software Zen2 and analyzed by custom ImageJ (v.1.51n). Statistical analyses were performed using GraphPad Prism 6.01 for MacOS. CIRCLE- CHANGE and IGUIDE analysis were done according to the respective publications and statements in the manuscript and were mapped to the following reference genomes: UCSC Macaca_fascicularis_5.0/macFas5; GRCh38/hg38; GRCm39/mm39. 
Policy information about availability of data

All manuscripts must include a data availability statement. This statement should provide the following information, where applicable:

- Accession codes, unique identifiers, or web links for publicly available datasets

- A list of figures that have associated raw data

- A description of any restrictions on data availability

The main data supporting the results is in the study are available within the paper and its Supplementary Information. The raw and analyzed dataasets generated during the study are too large to be publicly shared, yet they are available for research purposes from the corresponding authors on reasonable request. NGS data is publicly available (accession numbers: PRJEB41832 for WGS data, GSE168365 for NGS and RNA-seq data) Additionally used datasets in this study: UniVec (https:// www.ncbi.nlm.nih.gov/tools/vecscreen/univec/), refseq mRNA and genome sequences (human, mouse, arabidopsis, bacteria, virus, phix, lambda, mycoplasma: https://www.ncbi.nlm.nih.gov/refseq/), SILVA rRNA sequences (https://www.arb-silva.de/); Single Nucleotide Polymorphism Database (NCBI, v142),

\section{Field-specific reporting}

Please select the one below that is the best fit for your research. If you are not sure, read the appropriate sections before making your selection.

$\bigotimes$ Life sciences

Behavioural \& social sciences

Ecological, evolutionary \& environmental sciences

For a reference copy of the document with all sections, see nature.com/documents/nr-reporting-summary-flat.pdf

\section{Life sciences study design}

All studies must disclose on these points even when the disclosure is negative.

Sample size Sample sizes for in vivo experiments were determined based on literature precedence for genome editing experiments (Villiger 2018, Gaudelli, 2017, Koblan 2021). Further power calculation for treatment in the sensitized background was performed using G*Power (Faul, 2009) aiming for a confidence of $90 \%$ to assess a difference in HCC formation.

Data exclusions No animals and no data were excluded.

Replication All attempts at replication were successful. Findings were replicated as indicated in the figure legends.

Randomization Mice were assigned to the treated or untreated groups by litter. No covariates were controlled. For in vitro experiments tno samples were randomized.

\begin{tabular}{l|l} 
Blinding & Researchers were not blinded to group allocation. Blinding was not necessary because no placebo group was present and the readout (editing
\end{tabular} efficiency, PCSK9 levels, LDL levels) can not be influenced by a biased researcher. All HTS data was analyzed by an unblinded operator by using an automated script (CRISPResso2 + Javascript in Supplement) with limited experimenter intervention.

\section{Reporting for specific materials, systems and methods}

We require information from authors about some types of materials, experimental systems and methods used in many studies. Here, indicate whether each material, system or method listed is relevant to your study. If you are not sure if a list item applies to your research, read the appropriate section before selecting a response.

\begin{tabular}{|c|c|c|c|}
\hline \multicolumn{2}{|r|}{ Materials \& experimental systems } & \multicolumn{2}{|c|}{ Methods } \\
\hline$n / a$ & Involved in the study & $n / a$ & Involved in the study \\
\hline & $\bigotimes$ Antibodies & $\bigotimes$ & $\square$ ChIP-seq \\
\hline & Eukaryotic cell lines & $\bigotimes$ & $\square$ Flow cytometry \\
\hline$\lambda$ & $\square$ Palaeontology and archaeology & $\bigotimes$ & $\square$ MRI-based neuroimaging \\
\hline & Х Animals and other organisms & & \\
\hline$\bigotimes$ & $\square$ Human research participants & & \\
\hline Х & $\square$ Clinical data & & \\
\hline 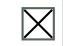 & $\square$ Dual use research of concern & & \\
\hline
\end{tabular}

\section{Antibodies}

Antibodies used

mouse-anti-Cas9 mAB (7A9-3A3; clone number:14697T, Cell Signaling, Cat. No\#14697); Goat-anti-mouse-HRP (SouthernBiotech, Cat. No. 1030-05) mouse-anti-monkey-HRP (SouthernBiotech, Cat. No. 4700-05); goat anti-Pcsk9 (1:10'000, Cat. No. AF3985-SP; R\&D Systems); rabbit anti-beta-actin (1:3’000, Cat. No. \#4970S; Cell Signaling) or rabbit anti-GAPDH (1:5’000, Cat. No. 4970; Abcam). HHRP- or IRDye-conjugated secondary antibodies (Donkey anti-Goat: Licor Cat. No. \#926-32214; anti-Rabbit: Licor Cat. No. \#926-68073 Cell Signaling Cat. No. \#7074, Promega Cat. No. \#V8051) 

Validation reference: Borys SM, Younger ST. Identification of functional regulatory elements in the human genome using pooled CRISPR screens. BMC Genomics. 2020 Jan 31;21(1):107. doi: 10.1186/s12864-020-6497-0. PMID: 32005150; PMCID: PMC6995077. Goat-anti-mouse-HRP: Validation: https://www.southernbiotech.com/?catno=1030-05\&type=Polyclonal\#\&panel1-1\&panel2-1. Validation reference: van Ginkel FW, Wahl SM, Kearney JF, Kweon M, Fujihashi K, Burrows PD, et al. Partial IgA-deficiency with increased Th2-type cytokines in TGF- $\beta 1$ knockout mice. J Immunol. 1999;163:1951-7. (ELISA, ELISPOT)

mouse-anti-monkey-HRP: Validation: https://www. southernbiotech.com/?catno=4700-05\&type=Monoclonal\#\&panel1-1. Validation reference: Kasturi SP, Skountzou I, Albrecht RA, Koutsonanos D, Hua T, Nakaya HI, et al. Programming the magnitude and persistence of antibody responses with innate immunity. Nature. 2011;470:543-7. (ELISA)

goat anti-Pcsk9: Validation: https://www.rndsystems.com/products/mouse-rat-proprotein-convertase-9-pcsk9-antibody_af3985. Validation reference: Kim K, Goldberg IJ, Graham MJ, Sundaram M, Bertaggia E, Lee SX, Qiang L, Haeusler RA, Metzger D, Chambon P, Yao Z, Ginsberg HN, Pajvani UB. $\gamma$-Secretase Inhibition Lowers Plasma Triglyceride-Rich Lipoproteins by Stabilizing the LDL Receptor. Cell Metab. 2018 Apr 3;27(4):816-827.e4. doi: 10.1016/j.cmet.2018.02.010. Epub 2018 Mar 22. PMID: 29576536; PMCID: PMC5884729.

rabbit anti-GAPDH: Validation: https://www.abcam.com/gapdh-antibody-epr16891-loading-control-ab181602.html. Validation reference: Gao Z et al. Regulatory effects of IncRNA ATB targeting miR-200c on proliferation and apoptosis of colorectal cancer cells. J Cell Biochem 121:332-343 (2020), PMID:31222825.

rabbit anti-beta-actin: Validation: https://www.cellsignal.com/products/primary-antibodies/b-actin-13e5-rabbit-mab/4970. Validation reference: Wang N, Yi H, Fang L, Jin J, Ma Q, Shen Y, Li J, Liang S, Xiong J, Li Z, Zeng H, Jiang F, Jin B, Chen L. CD226 Attenuates Treg Proliferation via Akt and Erk Signaling in an EAE Model. Front Immunol. 2020 Aug 21;11:1883. doi: 10.3389/ fimmu.2020.01883. PMID: 32983109; PMCID: PMC7478170.

\section{Eukaryotic cell lines}

Policy information about cell lines

Cell line source(s)

Hepa1-6 (ATCC CRL-18.30), HEK293T (ATCC CRL-321), HepG2 (ATCC HB-8065)

Authentication

Cell lines were authenticated by the supplier by STR analysis

Mycoplasma contamination

All cells were tested negative for mycoplasma

Commonly misidentified lines

(See ICLAC register)

No commonly misidentified cell lines were used

\section{Animals and other organisms}

Policy information about studies involving animals; ARRIVE guidelines recommended for reporting animal research

Laboratory animals

Wild animals

Field-collected samples

Ethics oversight
Male C57BL/6J mice (study start at 5 weeks of age, 20-25 g of weight)

Albumin-Cre x Trp53flox/flox mice, in C57BL/6J background (male and female (M/F) animals: Untreated (17/12); AAV only (16/2), ABE treated (16/9); study start at 5 weeks of age, $20-25 \mathrm{~g}$ of weight)

Mice were housed in a pathogen-free animal facility at the Institute of Molecular Health Sciences at ETH Zurich, and kept in a temperature- and humidity-controlled room on a 12 hours light-dark cycle.

Male Macaca fascicularis (approx. two years of age) were housed in a temperature- and humidity-controlled room on a 12 hours light-dark cycle.

The study did not involve wild animals

The study did not involve samples collected from the field

Mouse experiments were performed in accordance with protocols approved by the Kantonales Veterinäramt Zürich. Studies involving non-human primates were conducted at a facility accredited by the International Laboratory Animal Assessment and Accreditation (AAALAC), operating in accordance with the principles of US FDA's Good Laboratory Practice (GLP) and the Guide for the Care and Use of Laboratory Animals, Institute of Laboratory Animal Resources, National Academy Press, Washington, D.C., 2011. All protocols were reviewed and approved by the Acuitas animal care and use committee. 